\title{
$q$-Deformed superalgebras
}

\author{
Alexander Schmidt, Hartmut Wachter ${ }^{\dagger}$ \\ Max-Planck-Institute \\ for Mathematics in the Sciences \\ Inselstr. 22, D-04103 Leipzig, Germany \\ Arnold-Sommerfeld-Center \\ Ludwig-Maximilians-Universität \\ Theresienstr. 37, D-80333 München, Germany
}

\begin{abstract}
The article deals with $q$-analogs of the three- and four-dimensional Euclidean superalgebra and the Poincaré superalgebra.
\end{abstract}

*e-mail: schmidt@theorie.physik.uni-muenchen.de

${ }^{\dagger}$ e-mail: Hartmut.Wachter@physik.uni-muenchen.de 


\section{Introduction}

The idea of supersymmetry plays an important role in physics. Since its invention [1-7] a great experimental effort is undertaken to find supersymmetric particles in nature. The reason for this lies in the fact that supersymmetry could provide appealing solutions to outstanding problems in theoretical physics such as the so-called hierarchy problem. Furthermore, there are indications that supersymmetry can help in eliminating many of the divergences of certain quantum field theories. If supersymmetry becomes a local gauge symmetry it leads to supergravity, which is not as divergent as ordinary gravity $[8,9]$. Finally, the much-discussed superstring theories propose the existence of space-time supersymmetry.

Let us recall that supersymmetry extends the symmetry algebra of spacetime by adding supersymmetry generators. In the last two decades, however, a much more ambitious attempt to modify space-time symmetries has arisen. It is based on noncommutative geometry [10-15, 15-23] and tries to modify the whole space-time symmetry by deforming it in a consistent manner. There is a great hope that such an approach yields a discretization of space-time [24-29] which, in turn, implies an effective method for regularizing quantum field theories [30].

In our previous work [31-37] attention was concentrated on space-time structures that arise from $q$-deformation [38]. More concretely, we are interested in $q$-deformed quantum spaces that could prove useful in physical applications. For this reason we deal with the three- and four-dimensional $q$ deformed Euclidean space and the $q$-deformed Minkowski space [39-41]. The symmetries of these quantum spaces are described by the Drinfeld Jimbo algebras $U_{q}(s u(2))$ and $U_{q}(s o(4))$ [42-44] and the $q$-deformed Lorentz algebra [45]. Finally, we can combine these symmetry algebras with their quantum spaces and obtain $q$-analogs of the three- and four-dimensional Euclidean algebra and the Poincaré algebra [46,47].

It is an obvious thing to try to combine the ideas of supersymmetry with those of deforming space-time symmetries (see, for example, Refs. [4857]) and the aim of this article is to go the last step that makes the $q$ deformed Poincaré algebra and the $q$-deformed Euclidean algebras in three and four dimensions into superalgebras. To reach this goal we introduce supersymmetry generators with spinor indices and make suitable ansaetze for their commutation relations with the generators of the $q$-deformed Euclidean

algebras and the $q$-deformed Poincaré algebra. From consistency arguments 
we get a system of equations for the unknown coefficients of our ansaetze. Solving this system by a computer algebra system like Mathematica [58] we found that the relations of the three- and four-dimensional $q$-deformed Euclidean superalgebra are uniquely determined and the same holds for the $q$-deformed Poincaré superalgebra.

To write down our superalgebras in a rather compact form it is helpful to recognize adjoint actions as $q$-analogs of classical commutators. Using generators with definite transformation properties, these $q$-commutators can often be expressed by $q$-deformed Pauli matrices and their relatives (for their explicit form see Ref. [59]). Last but not least, it should be mentioned that in some sense the present article continues the reasonings of Ref. [60], where we adapted many general ideas about $q$-deformed quantum algebras to our framework of conventions and notations.

\section{Symmetry algebras for three-dimensional $q$-deformed Euclidean space}

In this section we first give a short review of the Hopf algebra $U_{q}(s u(2))$. Then, we combine this algebra with that of three-dimensional $q$-deformed momentum space and obtain a $q$-analog of the three-dimensional Euclidean algebra. These considerations culminate in the derivation of the threedimensional $q$-deformed Euclidean superalgebra.

\subsection{The Hopf algebra $U_{q}(s u(2))$}

The symmetry of $q$-deformed Euclidean space in three dimensions is described by the quantum algebra $U_{q}(s u(2))$ [41]. This algebra is spanned by the three generators $T^{+}, T^{-}$, and $T^{3}$ subject to the relations

$$
\begin{aligned}
& q^{-1} T^{+} T^{-}-q T^{-} T^{+}=T^{3}, \\
& q^{2} T^{3} T^{+}-q^{-2} T^{+} T^{3}=\lambda_{+} T^{+}, \\
& q^{-2} T^{3} T^{-}-q^{2} T^{-} T^{3}=-\lambda_{+} T^{-},
\end{aligned}
$$

where $\lambda_{+} \equiv q+q^{-1}$. Instead of working with $T^{3}$, it is often more convenient to use the generator $\tau^{3}$ defined by

$$
\tau^{3} \equiv 1-\lambda T^{3}, \quad \lambda \equiv q-q^{-1} .
$$


With this new generator the defining relations of $U_{q}(s l(2))$ become

$$
\begin{aligned}
T^{ \pm} \tau^{3} & =q^{ \pm 4} \tau^{3} T^{ \pm} \\
T^{-} T^{+} & =q^{-2} T^{+} T^{-}+q^{-1} \lambda^{-1}\left(\tau^{3}-1\right) .
\end{aligned}
$$

It is well-known that the algebra $U_{q}(s u(2))$ can be made into a Hopf algebra. The corresponding coproduct, antipode, and counit on its generators read as

$$
\begin{aligned}
\Delta\left(T^{ \pm}\right) & =T^{ \pm} \otimes 1+\left(\tau^{3}\right)^{1 / 2} \otimes T^{ \pm}, \\
\Delta\left(T^{3}\right) & =T^{3} \otimes 1+1 \otimes T^{3}-\lambda T^{3} \otimes T^{3}, \\
\Delta\left(\tau^{3}\right) & =\tau^{3} \otimes \tau^{3}, \\
S\left(T^{ \pm}\right) & =-\left(\tau^{3}\right)^{-1 / 2} T^{ \pm}, \\
S\left(T^{3}\right) & =-\left(\tau^{3}\right)^{-1} T^{3}, \\
S\left(\tau^{3}\right) & =\left(\tau^{3}\right)^{-1}, \\
\epsilon\left(T^{A}\right) & =0, \quad A \in\{+, 3,-\}, \\
\epsilon\left(\tau^{3}\right) & =1 .
\end{aligned}
$$

As symmetry algebra of three-dimensional q-deformed Euclidean space, $U_{q}(s u(2))$ can be viewed as $q$-analog of the algebra of three-dimensional angular momentum. This becomes more clear if we rewrite the defining relations of $U_{q}(s l(2))$ by means of the new generators

$$
\begin{aligned}
L^{ \pm} \equiv & -q^{ \pm 1 / 2} \lambda_{+}^{-1 / 2}\left(\tau^{3}\right)^{-1 / 2} T^{ \pm} \\
L^{3} \equiv & \left(\tau^{3}\right)^{1 / 2}\left(L^{-} L^{+}-L^{+} L^{-}\right) \\
= & \lambda^{-1} \lambda_{+}^{-1}\left(\left(\tau^{3}\right)^{-1 / 2} T^{-} T^{+}-q^{-1}\left(\tau^{3}\right)^{-1 / 2}\right. \\
& \left.+q^{-1}\left(\tau^{3}\right)^{1 / 2}+2 q\right) .
\end{aligned}
$$

In this manner, we get

$$
\begin{aligned}
L^{ \pm} \tau^{3} & =q^{ \pm 4} \tau^{3} L^{ \pm}, \quad L^{3} \tau^{3}=\tau^{3} L^{3} \\
L^{-} L^{+} & =q^{2} L^{+} L^{-}+q \lambda^{-1} \lambda_{+}^{-1}\left(\left(\tau^{3}\right)^{-1}-1\right),
\end{aligned}
$$

or, alternatively,

$$
L^{-} L^{+}-L^{+} L^{-}=\left(\tau^{3}\right)^{-1 / 2} L^{3}
$$




$$
L^{ \pm} L^{3}-L^{3} L^{ \pm}=q^{ \pm 1} L^{ \pm}\left(\tau^{3}\right)^{-1 / 2}
$$

In the classical limit $q \rightarrow 1$ we regain the Lie-algebra $s u(2)$ from the relations in (9). This can easily be seen if we recognize that $\tau^{3}$ tends to 1 for $q \rightarrow 1$. Thus, $L^{+}, L^{3}$, and $L^{-}$play the role of the components of $q$-deformed angular momentum in three dimensions. Their coproducts, antipodes, and counits are found to be

$$
\begin{aligned}
\Delta\left(L^{ \pm}\right)= & L^{ \pm} \otimes\left(\tau^{3}\right)^{-1 / 2}+1 \otimes L^{ \pm}, \\
\Delta\left(L^{3}\right)= & L^{3} \otimes\left(\tau^{3}\right)^{-1 / 2}+\left(\tau^{3}\right)^{1 / 2} \otimes L^{3}, \\
& +\lambda\left(\tau^{3}\right)^{1 / 2}\left(q^{-1} L^{-} \otimes L^{+}+q L^{+} \otimes L^{-}\right), \\
S\left(L^{ \pm}\right)= & -L^{ \pm}\left(\tau^{3}\right)^{1 / 2}, \\
S\left(L^{3}\right)= & \left(\tau^{3}\right)^{3 / 2}\left(q^{2} L^{+} L^{-}-q^{-2} L^{-} L^{+}\right), \\
\epsilon\left(L^{A}\right)= & 0 \quad A \in\{+, 3,-\} .
\end{aligned}
$$

In terms of the generators of $q$-deformed angular momentum, the Casimir operator of $U_{q}(s u(2))$ takes on the rather intuitive form [60]

$$
L^{2} \equiv g_{A B} L^{A} L^{B}=-q L^{+} L^{-}+L^{3} L^{3}-q^{-1} L^{-} L^{+},
$$

where $g_{A B}$ denotes the quantum metric of three-dimensional q-deformed Euclidean space. Notice that repeated indices are to be summed over if not stated otherwise.

It should also be noted that the components of three-dimensional angular momentum give rise to a quantum Lie algebra. To this end we introduce $q$-analogs of classical commutators. These so-called $q$-commutators are determined by the Hopf structure of $U_{q}(s u(2))$ [60,64-66]:

$$
\left[L^{A}, L^{B}\right]_{q} \equiv L_{(1)}^{A} L^{B} S\left(L_{(2)}^{A}\right)=S^{-1}\left(L_{(2)}^{B}\right) L^{A} L_{(1)}^{B},
$$

where we have written the coproduct in Sweedler notation. It is now straightforward to check that the relations in (9) are equivalent to [60]:

$$
\left[L^{A}, L^{B}\right]_{q}=q^{2} \varepsilon^{A B C} g_{C D} L^{D}
$$

where $\varepsilon^{A B C}$ denotes the three-dimensional $q$-deformed epsilon tensor (its nonvanishing components are listed in the appendix). 
The components $L^{+}, L^{3}$, and $L^{-}$transform under $U_{q}(s u(2))$ as a vector. Using the three-dimensional $q$-deformed epsilon tensor we are able to assign the components of angular momentum an antisymmetric tensor $M^{A B}$ [60]:

$$
M^{A B} \equiv k_{1} \varepsilon^{A B C} g_{C D} L^{D} .
$$

More explicitly, we have

$$
\begin{gathered}
M^{+3}=-k_{1} q^{-1} L^{+}, \quad M^{3-}=-k_{1} q^{-1} L^{-}, \\
M^{+-}=\lambda M^{33}=-k_{1} q^{-2} L^{3} .
\end{gathered}
$$

We regain the components of three-dimensional angular momentum through

$$
L^{D}=k_{1}^{\prime} g^{D C} \varepsilon_{B A C} M^{A B},
$$

being tantamount to

$$
\begin{aligned}
L^{+} & =-k_{1}^{\prime} q^{-3}\left(q^{2}+q^{-2}\right) M^{+3}, \\
L^{3} & =-k_{1}^{\prime} q^{-2}\left(q^{2}+q^{-2}\right) M^{+-}, \\
L^{-} & =-k_{1}^{\prime} q^{-3}\left(q^{2}+q^{-2}\right) M^{3-},
\end{aligned}
$$

where our choice of conventions requires that

$$
k_{1} k_{1}^{\prime}=q^{4}\left(q^{2}+q^{-2}\right)^{-1} .
$$

Using the generators in (16) the Casimir in (13) reads as

$$
L^{2}=\frac{\left(k_{1}^{\prime}\right)^{2} q^{4}}{q^{2}+q^{-2}} g_{A D} g_{B C} M^{A B} M^{C D} .
$$

In the same manner, the relations for the quantum Lie algebra of $U_{q}(s u(2))$ turn into

$$
\left[M^{A B}, M^{C D}\right]_{q}=k^{\prime-1} \frac{q^{2}\left(q^{2}+q^{-2}\right)}{q^{2}-1+q^{-2}}\left(P_{A}\right){ }_{E F}^{A B}\left(P_{A}\right){ }_{G H}^{C D} g^{F G} M^{E H},
$$

or, explicitly,

$$
\begin{aligned}
{\left[M^{+3}, M^{+-}\right]_{q} } & =-q^{-2}\left[M^{+-}, M^{+3}\right]_{q}=k^{\prime-1} q^{7}\left(q^{2}+q^{-2}\right)^{-1} M^{+3} \\
{\left[M^{+3}, M^{3-}\right]_{q} } & =-\left[M^{3-}, M^{+3}\right]_{q}=k^{\prime-1} q^{8}\left(q^{2}+q^{-2}\right)^{-1} M^{+-} \\
{\left[M^{+-}, M^{3-}\right]_{q} } & =-q^{-2}\left[M^{3-}, M^{+-}\right]_{q}=k^{\prime-1} q^{7}\left(q^{2}+q^{-2}\right)^{-1} M^{3-} .
\end{aligned}
$$

Notice that in analogy to (14) we have

$$
\left[M^{A B}, M^{C D}\right]_{q} \equiv M_{(1)}^{A B} M^{C D} S\left(M_{(2)}^{A B}\right)=S^{-1}\left(M_{(2)}^{C D}\right) M^{A B} M_{(1)}^{C D} .
$$




\subsection{The three-dimensional $q$-deformed Euclidean alge- bra}

Next, we wish to combine the algebra of three-dimensional angular momentum with the algebra of $q$-deformed momentum space. This way, we arrive at a $q$-analog of the three-dimensional $q$-deformed Euclidean algebra. First of all, let us recall the commutation relations for the three-dimensional momentum generators $P^{A}, A \in\{+, 3,-\}$ :

$$
\left(P_{A}\right)^{A B}{ }_{C D} P^{A} P^{B}=0,
$$

where $P_{A}$ stands for the antisymmetrizer of three-dimensional $q$-deformed Euclidean space [41]. The above condition implies as independent relations

$$
P^{3} P^{ \pm}-q^{ \pm 2} P^{ \pm} P^{3}=0, \quad P^{-} P^{+}-P^{+} P^{-}=\lambda P^{3} P^{3} .
$$

It remains to specify the commutation relations between the generators of $U_{q}(s u(2))$ and the momentum algebra. To this end, we first have to realize that the momentum generators establish a vector representation of $U_{q}(s u(2))$. The point now is that we can combine a Hopf algebra $\mathcal{H}$ with its representation space $\mathcal{A}$ to form a so-called left-cross product algebra $\mathcal{A} \rtimes \mathcal{H}$ [61-63] built on $\mathcal{A} \otimes \mathcal{H}$ with product

$$
(a \otimes h)(b \otimes g)=a\left(h_{(1)} \triangleright b\right) \otimes h_{(2)} g, \quad a, b \in \mathcal{A}, \quad h, g \in \mathcal{H} .
$$

The last identity tell us that the commutation relations between symmetry generators and representation space elements are completely determined by coproduct and action of the Hopf algebra $\mathcal{H}$, since we have

$$
h b=(1 \otimes h)(b \otimes 1)=\left(h_{(1)} \triangleright b\right) \otimes h_{(2)} .
$$

Applying these ideas to $U_{q}(s u(2))$ and the three-dimensional $q$-deformed momentum algebra we obtain the relations

$$
\begin{gathered}
L^{ \pm} P^{ \pm}-P^{ \pm} L^{ \pm}=0, \\
L^{ \pm} P^{\mp}-P^{\mp} L^{ \pm}=\mp P^{3} \tau^{-1 / 2}, \\
L^{ \pm} P^{3}-P^{3} L^{ \pm}=\mp q^{ \pm 1} P^{ \pm} \tau^{-1 / 2}, \\
L^{3} P^{ \pm}-q^{\mp 2} P^{ \pm} L^{3}= \pm q^{\mp 1} \lambda P^{3} L^{ \pm} \pm q^{\mp 1} P^{ \pm} \tau^{-1 / 2}, \\
L^{3} P^{3}-P^{3} L^{3}=\lambda\left(P^{-} L^{+}-P^{+} L^{-}\right)-\lambda P^{3} \tau^{-1 / 2} .
\end{gathered}
$$


By means of the $q$-commutator

$$
\left[L^{A}, P^{B}\right]_{q} \equiv L^{A} \triangleright P^{B}=L_{(1)}^{A} P^{B} S\left(L_{(2)}^{A}\right),
$$

which is nothing other than the adjoint action of $L^{A}$ on $P^{B}$, the relations in (29) can be written more compactly:

$$
\left[L^{A}, P^{B}\right]_{q}=q^{2} \varepsilon^{A B C} g_{C D} P^{D} .
$$

Last but not least, we would like to give the Casimir operators of the three-dimensional q-deformed Euclidean algebra. With the commutation relations presented so far one can verify that the operators

$$
C_{1} \equiv g_{A B} P^{A} P^{B}, \quad C_{2} \equiv g_{A B} L^{A} P^{B}
$$

commute with all generators of $U_{q}(s u(2))$ and the momentum algebra.

\subsection{Symmetry algebra in spinor notation}

In Ref. [59] we discussed $q$-analogs of Pauli matrices that enable us to construct a vector out of two spinors. On these grounds, we can use $q$-deformed Pauli matrices to switch from the vectorial generators $L^{\mu}$ to operators $M^{\alpha \beta}$ with two spinor indices:

$$
M^{\alpha \beta}=k_{2}^{\prime}\left(\sigma_{A}^{-1}\right)^{\alpha \beta} L^{A} .
$$

From the completeness relation

$$
\left(\sigma^{A}\right)_{\alpha \beta}\left(\sigma_{B}^{-1}\right)^{\alpha \beta}=\delta_{B}^{A}
$$

we get, at once,

$$
L^{A}=k_{2}\left(\sigma^{A}\right)_{\alpha \beta} M^{\alpha \beta},
$$

with $k_{2} k_{2}^{\prime}=1$. Explicitly, we have

$$
\begin{aligned}
M^{11}=k_{2}^{\prime} q^{-1} L^{-}, & M^{22}=k_{2}^{\prime} q^{-1} L^{+}, \\
M^{12}=k_{2}^{\prime} q^{-1 / 2} \lambda_{+}^{-1 / 2} L^{3}, & M^{21}=k_{2}^{\prime} q^{-3 / 2} \lambda_{+}^{-1 / 2} L^{3},
\end{aligned}
$$

and

$$
L^{+}=k_{2} q M^{22}, \quad L^{-}=k_{2} q M^{11},
$$




$$
\begin{aligned}
L^{3} & =k_{2} q \lambda_{+}^{-1 / 2}\left(q^{1 / 2} M^{12}+q^{-1 / 2} M^{21}\right) \\
& =k_{2} q^{1 / 2} \lambda_{+}^{1 / 2} M^{12} .
\end{aligned}
$$

The matrix entries of the Pauli matrices $\sigma^{A}$ and $\sigma_{A}^{-1}$ can be looked up in Ref. [59]. It should also be mentioned that the so-called 'inverse' Pauli matrices $\sigma_{A}^{-1}$ should not be confused with matrices being inverse in the sense of matrix multiplication (for the details see again Ref. [59]). Especially, we have the identification

$$
\left(\sigma_{A}^{-1}\right)^{\alpha \beta}=q^{-2}\left(\sigma^{A}\right)_{\alpha \beta} \quad A=\{+, 3,-\} .
$$

We can also start our considerations from the generators $M^{A B}$ introduced in (16). They are related to the generators $M^{\alpha \beta}$ by the formulae

$$
M^{\alpha \beta}=k_{3}^{\prime}\left(\sigma_{A B}^{-1}\right)^{\alpha \beta} M^{A B}, \quad M^{A B}=k_{3}\left(\sigma^{A B}\right)_{\alpha \beta} M^{\alpha \beta},
$$

where $\sigma^{A B}$ and $\sigma_{A B}^{-1}$ denote the two-dimensional spin matrices of threedimensional $q$-deformed Euclidean space. For their explicit form we refer the reader again to Ref. [59]. Written out explicitly, the relations in (39) become

$$
\begin{gathered}
M^{11}=k_{3}^{\prime} q^{-1} \lambda_{+}^{-1 / 2} M^{3-}, \quad M^{22}=k_{3}^{\prime} q^{-1} \lambda_{+}^{-1 / 2} M^{+3} \\
M^{12}=q M^{21}=k_{3}^{\prime} q^{-3 / 2} \lambda_{+}^{-1} M^{+-}
\end{gathered}
$$

and

$$
\begin{gathered}
M^{+3}=k_{3} q^{3} \lambda_{+}^{-1 / 2} M^{22}, \quad M^{3-}=k_{3} q^{3} \lambda_{+}^{-1 / 2} M^{11}, \\
M^{+-}=\lambda M^{33}=k_{3} q^{3 / 2} M^{12}
\end{gathered}
$$

The two-dimensional spin matrices are subject to the completeness relation

$$
\left(\sigma^{A B}\right)_{\alpha \beta}\left(\sigma_{C D}^{-1}\right)^{\alpha \beta}=q^{-2}\left(q^{4}+1\right) \lambda_{+}^{-1}\left(P_{A}\right)^{A B}{ }_{C D} .
$$

This relation implies

$$
k_{3} k_{3}^{\prime}=q^{2}\left(q^{4}+1\right)^{-1} \lambda_{+}^{-1},
$$

as can be proven by inserting the two equations of (39) into each other. Finally, it should be mentioned that the relations in (39) are consistent with (16) and (18) iff the following condition is satisfied:

$$
k_{2}^{\prime} k_{3}=-q^{3} \lambda_{+}^{-1 / 2} k_{1} .
$$


Last but not least, we use the generators $M^{\alpha \beta}$ with two spinor indices to write the quantum Lie algebra and the Casimir operator of $U_{q}(s u(2))$ in an alternative form. Clearly, the $q$-commutators between the $M^{\alpha \beta}$ are defined in complete analogy to (24). Using the correspondence between the $M^{\alpha \beta}$ and the vectorial generators $L^{A}$ one can show that

$$
\begin{aligned}
& {\left[M^{12}, M^{12}\right]_{q}=-k_{2}^{-1} q^{-1 / 2} \lambda \lambda_{+}^{-1 / 2} M^{12},} \\
& {\left[M^{11}, M^{12}\right]_{q}=-q^{-2}\left[M^{12}, M^{11}\right]_{q}=k_{2}^{-1} q^{-3 / 2} \lambda_{+}^{-1 / 2} M^{11},} \\
& {\left[M^{11}, M^{22}\right]_{q}=-\left[M^{22}, M^{11}\right]_{q}=k_{2}^{-1} q^{-3 / 2} \lambda_{+}^{1 / 2} M^{12},} \\
& {\left[M^{12}, M^{22}\right]_{q}=-q^{-2}\left[M^{22}, M^{12}\right]_{q}=k_{2}^{-1} q^{-3 / 2} \lambda_{+}^{-1 / 2} M^{22},}
\end{aligned}
$$

and the remaining $q$-commutators all vanish. Inserting (37) in (13) we find the following expression for the Casimir operator of $U_{q}(s u(2))$ :

$$
C=k_{2}^{2}\left(-q^{3} M^{11} M^{22}-q M^{22} M^{11}+q \lambda_{+} M^{12} M^{12}\right) .
$$

The $q$-commutators in (45) and the Casimir (46) can be written in a closed form by using the $q$-deformed spinor metric $\varepsilon_{\alpha \beta}$ and the symmetric projector $S$ corresponding to the $\hat{R}$-matrix of $U_{q}(s u(2))$. In this manner, we have

$$
\begin{aligned}
{\left[M^{\alpha \beta}, M^{\gamma \delta}\right]_{q} } & =-k_{2}^{-1} q^{-1} \lambda_{+}^{1 / 2} S_{\beta^{\prime} \alpha^{\prime}}^{\alpha \beta} S_{\delta^{\prime} \gamma^{\prime}}^{\gamma \delta} \varepsilon^{\alpha^{\prime} \delta^{\prime}} M^{\beta^{\prime} \gamma^{\prime}} \\
C & =-k_{2}^{2} q^{2} \varepsilon_{\alpha \beta} \varepsilon_{\alpha^{\prime} \beta^{\prime}} M^{\alpha^{\prime} \alpha} M^{\beta \beta^{\prime}} .
\end{aligned}
$$

\subsection{The three-dimensional $q$-deformed Euclidean su- peralgebra}

In the previous sections we considered the $q$-deformed Euclidean algebra in three dimensions. This algebra is a cross product of $U_{q}(s u(2))$ and threedimensional $q$-deformed Euclidean space. In this sense, it is spanned by the generators of $U_{q}(s u(2))$ and the components of three-dimensional $q$-deformed momentum.

For implementing supersymmetry on $q$-deformed Euclidean space in three dimensions, one has to extend its Euclidean algebra to a $q$-deformed Euclidean superalgebra. To describe a $q$-deformed version of $N=1$ supersymmetry we have to add supersymmetry generators $Q^{\alpha}$ and $\bar{Q}^{\alpha}$ with spinor indices. It is now our task to determine the commutation relations concerning the new generators. 
We assume that $Q^{1}$ together with $Q^{2}$ generate an antisymmetrized quantum plane and the same should hold for $\bar{Q}^{1}$ and $\bar{Q}^{2}$. Thus, we have

$$
\begin{aligned}
& Q^{\alpha} Q^{\beta}=-q \hat{R}^{\alpha \beta}{ }_{\alpha^{\prime} \beta^{\prime}} Q^{\alpha^{\prime}} Q^{\beta^{\prime}}, \\
& \bar{Q}^{\alpha} \bar{Q}^{\beta}=-q \hat{R}^{\alpha \beta}{ }_{\alpha^{\prime} \beta^{\prime}} \bar{Q}^{\alpha^{\prime}} \bar{Q}^{\beta^{\prime}},
\end{aligned}
$$

where $\hat{R}$ stands for the $\hat{R}$-matrix of $U_{q}(s u(2))$. From (48) we find as independent relations

$$
\begin{gathered}
Q^{\alpha} Q^{\alpha}=\bar{Q}^{\dot{\alpha}} \bar{Q}^{\dot{\alpha}}=0, \\
Q^{1} Q^{2}=-q^{-1} Q^{2} Q^{1}, \quad \bar{Q}^{1} \bar{Q}^{2}=-q^{-1} \bar{Q}^{2} \bar{Q}^{1} .
\end{gathered}
$$

If we introduce a $q$-deformed anticommutator for spinor operators by

$$
\left\{\theta^{\alpha}, \tilde{\theta}^{\beta}\right\}_{k} \equiv \tilde{\theta}^{\alpha} \theta^{\beta}+k \hat{R}_{\alpha^{\prime} \beta^{\prime}}^{\alpha \beta} \theta^{\alpha^{\prime}} \tilde{\theta}^{\beta^{\prime}},
$$

the relations in (48) become

$$
\left\{Q^{\alpha}, Q^{\beta}\right\}_{q}=\left\{\bar{Q}^{\alpha}, \bar{Q}^{\beta}\right\}_{q}=0 .
$$

It is obvious that $Q^{\alpha}$ as well as $\bar{Q}^{\alpha}$ establish spin-1/2 representations of $U_{q}(s u(2))$. This observation fixes the commutation relations of the supersymmetry generators with the generators of $U_{q}(s u(2))$. As it was shown in Ref. [60] the spinor operators $Q^{\alpha}$ and $\bar{Q}^{\alpha}$ then have to fulfill

$$
\begin{aligned}
& {\left[L^{A}, Q^{\alpha}\right]_{q}=-q^{-1} \lambda_{+}^{-1 / 2}\left(\sigma^{A}\right)_{\beta}{ }^{\alpha} Q^{\beta},} \\
& {\left[L^{A}, \bar{Q}^{\alpha}\right]_{q}=-q^{-1} \lambda_{+}^{-1 / 2}\left(\sigma^{A}\right)_{\beta}{ }^{\alpha} \bar{Q}^{\beta},}
\end{aligned}
$$

where the $q$-commutator is a kind of shorthand notation for the adjoint action, i.e.

$$
\left[L^{A}, V\right]_{q} \equiv L_{(1)}^{A} V S\left(L_{(2)}^{A}\right) .
$$

Notice that in (52) we used the Pauli matrices $\sigma^{A}$ given in Ref. [59]. If we instead work with the generators (17) or (36) we respectively have

$$
\begin{aligned}
& {\left[M^{A B}, Q^{\alpha}\right]_{q}=\left(k_{1}^{\prime}\right)^{-1} q^{4}\left(q^{2}+q^{-2}\right)^{-1} Q^{\beta}\left(\sigma^{A B}\right)_{\beta}{ }^{\alpha},} \\
& {\left[M^{A B}, \bar{Q}^{\alpha}\right]_{q}=\left(k_{1}^{\prime}\right)^{-1} q^{4}\left(q^{2}+q^{-2}\right)^{-1} \bar{Q}^{\beta}\left(\sigma^{A B}\right)_{\beta}{ }^{\alpha},}
\end{aligned}
$$

and

$$
\left[M^{\alpha \beta}, Q^{\gamma}\right]_{q}=-k_{2}^{-1} q^{-1} \lambda_{+}^{-1 / 2} S_{\alpha^{\prime} \beta^{\prime}}^{\alpha \beta} \varepsilon^{\beta^{\prime} \gamma} Q^{\alpha^{\prime}}
$$




$$
\left[M^{\alpha \beta}, \bar{Q}^{\gamma}\right]_{q}=-k_{2}^{-1} q^{-1} \lambda_{+}^{-1 / 2} S_{\alpha^{\prime} \beta^{\prime}}^{\alpha \beta} \varepsilon^{\beta^{\prime} \gamma} \bar{Q}^{\alpha^{\prime}}
$$

It remains to find the commutation relations between $Q^{\alpha}$ and $\bar{Q}^{\alpha}$. In addition to this, we have to specify how the momentum components $P^{A}$ commute with the supersymmetry generators. To this end, one can make reasonable ansaetze for the wanted commutation relations. First of all, they are restricted by the requirement that the commutation relations have to be covariant with respect to the action of $U_{q}(s u(2))$. In addition to this, the commutation relations should define an ideal of the algebra generated by $Q^{\alpha}, \bar{Q}^{\alpha}, P^{A}$, and the generators of $U_{q}(s u(2))$. In other words, multiplying a relation by a generator and commuting this generator to the other side of the relation must not change the relation. This kind of consistency condition completely determines the commutation relations between the $Q^{\alpha}, \bar{Q}^{\alpha}$, and $P^{A}$.

This way, we found for the commutation relations between $Q^{\alpha}$ and $\bar{Q}^{\alpha}$ that

$$
\begin{aligned}
\bar{Q}^{1} Q^{1}+Q^{1} \bar{Q}^{1} & =q^{1 / 2} \lambda_{+}^{1 / 2} c P^{-}, \\
\bar{Q}^{1} Q^{2}+q^{-1} Q^{2} \bar{Q}^{1} & =-q^{-1} \lambda Q^{1} \bar{Q}^{2}+q c P^{3} \\
\bar{Q}^{2} Q^{1}+q^{-1} Q^{1} \bar{Q}^{2} & =c P^{3} \\
\bar{Q}^{2} Q^{2}+Q^{2} \bar{Q}^{2} & =q^{1 / 2} \lambda_{+}^{1 / 2} c P^{+}
\end{aligned}
$$

where the constant $c$ remains undetermined. We are free to choose $c=1$. The commutation relations between $P^{A}$ and $Q^{\alpha}$ take the form

$$
\begin{aligned}
P^{+} Q^{1} & =q^{-2} Q^{1} P^{+}, \\
P^{+} Q^{2} & =Q^{2} P^{+}, \\
P^{3} Q^{1} & =q^{-1} Q^{1} P^{3}, \\
P^{3} Q^{2} & =q^{-1} Q^{2} P^{3}+q^{-3 / 2} \lambda \lambda_{+}^{1 / 2} Q^{1} P^{+}, \\
P^{-} Q^{1} & =Q^{1} P^{-}, \\
P^{-} Q^{2} & =q^{-2} Q^{2} P^{-}+q^{-3 / 2} \lambda \lambda_{+}^{1 / 2} Q^{1} P^{3} .
\end{aligned}
$$

Likewise, we have

$$
\begin{aligned}
& P^{-} \bar{Q}^{1}=\bar{Q}^{1} P^{-} \\
& P^{-} \bar{Q}^{2}=q^{2} \bar{Q}^{2} P^{-} \\
& P^{3} \bar{Q}^{1}=q \bar{Q}^{1} P^{3}-q^{3 / 2} \lambda \lambda_{+}^{1 / 2} \bar{Q}^{2} P^{-}
\end{aligned}
$$




$$
\begin{aligned}
& P^{3} \bar{Q}^{2}=q \bar{Q}^{2} P^{3} \\
& P^{+} \bar{Q}^{1}=q^{2} \bar{Q}^{1} P^{+}-q^{3 / 2} \lambda \lambda_{+}^{1 / 2} \bar{Q}^{2} P^{3} \\
& P^{+} \bar{Q}^{2}=\bar{Q}^{2} P^{+}
\end{aligned}
$$

Again, the relations in (ㅎ6)-( of $q$-commutators and $q$-anticommutators. In the case of (56), for example, one checks that

$$
\left\{\bar{Q}^{\alpha}, Q^{\beta}\right\}_{q^{-1}}=\bar{Q}^{\alpha} Q^{\beta}+q^{-1} \hat{R}_{\alpha^{\prime} \beta^{\prime}}^{\alpha \beta} Q^{\alpha^{\prime}} \bar{Q}^{\beta^{\prime}}=c q^{1 / 2} \lambda_{+}^{-1 / 2}\left(\sigma_{A}^{-1}\right)^{\alpha \beta} P^{A} .
$$

To find an analogous form for the relations in (157) and (58) we define [67]

$$
\left[P^{\mu}, V\right]_{q} \equiv P_{(1)}^{\mu} V S\left(P_{(2)}^{\mu}\right), \quad\left[P^{\mu}, V\right]_{\bar{q}} \equiv P_{(\overline{1})}^{\mu} V \bar{S}\left(P_{(\overline{2})}^{\mu}\right)
$$

The calculation of these $q$-commutators requires to know the Hopf structures for the momentum algebra. The corresponding coproducts, antipodes, and counits on the momentum generators read as

$$
\begin{aligned}
\Delta\left(P^{A}\right) & =P_{(1)}^{A} \otimes P_{(2)}^{A}=P^{A} \otimes 1+\mathcal{L}^{A}{ }_{B} \otimes P^{B} \\
\bar{\Delta}\left(P^{A}\right) & =P_{(\overline{1})}^{A} \otimes P_{(\overline{2})}^{A}=P^{A} \otimes 1+\overline{\mathcal{L}}^{A}{ }_{B} \otimes P^{B}, \\
S\left(P^{A}\right) & =-S\left(\mathcal{L}^{A}{ }_{B}\right) P^{B}, \\
\bar{S}\left(P^{A}\right) & =-S\left(\overline{\mathcal{L}}^{A}{ }_{B}\right) P^{B}, \\
\epsilon\left(P^{A}\right) & =\bar{\epsilon}\left(P^{A}\right)=0 .
\end{aligned}
$$

Notice that $\mathcal{L}$ and $\overline{\mathcal{L}}$ respectively stand for the $L$-matrix and its conjugate. Their explicit form can be found in Refs. [32,35]. It should also be mentioned that these $L$-matrices realize the generators of the quantum group $S U_{q}(2)$ within the algebra $U_{q}(s u(2))$. On these grounds, they depend on generators of $U_{q}(s u(2))$ and a unitary scaling operator $\Lambda$ subject to

$$
\Lambda Q^{\alpha}=q^{2} Q^{\alpha} \Lambda, \quad \Lambda \bar{Q}^{\alpha}=q^{2} \bar{Q}^{\alpha} \Lambda
$$

Let us now collect all relations of the three-dimensional q-deformed Euclidean superalgebra:

$$
\begin{aligned}
& {\left[L^{A}, L^{B}\right]_{q}=q^{2} \varepsilon^{A B C} g_{C D} L^{D}} \\
& {\left[L^{A}, P^{B}\right]_{q}=q^{2} \varepsilon^{A B C} g_{C D} P^{D}}
\end{aligned}
$$




$$
\begin{gathered}
\left(P_{A}\right)^{A B}{ }_{C D} P^{A} P^{B}=0, \\
{\left[L^{A}, Q^{\alpha}\right]_{q}=-q^{-1} \lambda_{+}^{-1 / 2}\left(\sigma^{A}\right)_{\beta}{ }^{\alpha} Q^{\beta},} \\
{\left[L^{A}, \bar{Q}^{\alpha}\right]_{q}=-q^{-1} \lambda_{+}^{-1 / 2}\left(\sigma^{A}\right)_{\beta}{ }^{\alpha} \bar{Q}^{\beta},} \\
{\left[P^{A}, Q^{\alpha}\right]_{\bar{q}}=0, \quad\left[P^{A}, \bar{Q}^{\alpha}\right]_{q}=0,} \\
\left\{Q^{\alpha}, Q^{\beta}\right\}_{q}=0, \quad\left\{\bar{Q}^{\alpha}, \bar{Q}^{\beta}\right\}_{q}=0, \\
\left\{\bar{Q}^{\alpha}, Q^{\beta}\right\}_{q^{-1}}=q^{3 / 2} \lambda_{+}^{1 / 2}\left(\sigma_{A}^{-1}\right)^{\alpha \beta} P^{A} .
\end{gathered}
$$

We would like to end this section with some remarks about this algebra. First of all, the reader should be aware of the fact that $U_{q}(s u(2))$ cannot be generated by $L^{+}, L^{3}$, and $L^{-}$alone. For this reason we have to add the grouplike operator $\tau^{3}$ and take attention of its commutation relations with the generators $Q^{\alpha}, \bar{Q}^{\alpha}$, and $P^{A}$ :

$$
\begin{array}{rlrl}
\tau^{3} P^{ \pm} & =q^{\mp 4} P^{ \pm} \tau^{3}, & \tau^{3} P^{3}=P^{3} \tau^{3}, \\
\tau^{3} Q^{1} & =q^{2} Q^{1} \tau^{3}, & \tau^{3} Q^{2} & =q^{-2} Q^{2} \tau^{3}, \\
\tau^{3} Q^{1} & =q^{2} Q^{1} \tau^{3}, & \tau^{3} Q^{2} & =q^{-2} Q^{2} \tau^{3} .
\end{array}
$$

In the form of (63) the $q$-deformed superalgebra is strongly reminiscent of its classical counterpart, to which it tends if $q \rightarrow 1$. Notice that in the undeformed limit the $q$-commutators and $q$-anticommutators then pass into ordinary commutators and anticommutators, respectively.

It should also be mentioned that the algebra in (63) is compatible with the conjugation assignment

$$
\begin{gathered}
\overline{L^{A}}=g_{A B} L^{B}, \quad \overline{\tau^{3}}=\tau^{3}, \quad \overline{P^{A}}=g_{A B} P^{B}, \\
\overline{Q^{\alpha}}=\varepsilon_{\alpha \beta} \bar{Q}^{\beta}, \quad \overline{\bar{Q}^{\alpha}}=-\varepsilon_{\alpha \beta} Q^{\beta} .
\end{gathered}
$$

Indeed, one can check that conjugating the relations of our superalgebra and applying (65) does not change the relations in (63). In this sense, the three-dimensional $q$-deformed Euclidean superalgebra is real.

From Ref. [59] we know of different types of $q$-deformed Pauli and spin matrices. In this section we used matrices for symmetrized spinors, but nothing prevents us to work with matrices for antisymmetrized spinors. For the details we refer the reader to Ref. [59]. 


\section{Symmetry algebras for four-dimensional $q$ - deformed Euclidean space}

The consideration for the three-dimensional $q$-deformed Euclidean space carry over to the four-dimensional one. For this reason, we can restrict ourselves to stating the results, only.

\subsection{The four-dimensional $q$-deformed Euclidean alge- bra}

The symmetry of four-dimensional $q$-deformed Euclidean space is described by the quantum algebra $U_{q}(s o(4))$. This algebra can be viewed as tensor product of two commuting copies of $U_{q}(s u(2))$, i.e.

$$
U_{q}(s o(4)) \cong U_{q}(s u(2)) \otimes U_{q}(s u(2)) .
$$

The two sets of $U_{q}(s u(2))$-generators are denoted by $L_{i}^{ \pm}, K_{i}, i=1,2$, where the $L_{i}^{ \pm}$play the role of $T^{ \pm}$and the $K_{i}$ that of $\left(\tau^{3}\right)^{-1 / 2}$. Thus, the commutation relations between generators of the same lower index read [68]

$$
\begin{gathered}
q^{-1} L_{i}^{+} L_{i}^{-}-q L_{i}^{-} L_{i}^{+}=\lambda^{-1}\left(1-K_{i}^{-2}\right), \\
L_{i}^{ \pm} K_{i}=q^{\mp 2} K_{i} L_{i}^{ \pm}, \quad i=1,2,
\end{gathered}
$$

whereas generators with different lower indices always commute.

The generators of $U_{q}(s o(4))$ can be related to the components $L^{\mu \nu}, \mu, \nu=$ $1, \ldots, 4$, of an antisymmetric tensor operator (see, for example, Ref. [68]). In Ref. [60] we saw that the $L^{\mu \nu}$ give rise to a quantum Lie algebra of $U_{q}(s o(4) \dot{)}$. The explicit form of the corresponding $q$-commutators can be found in the work of Ref. [60]. Here, we only give the general expression

$$
\left[L^{\mu \nu}, L^{\rho \sigma}\right]_{q}=-q^{-1} \lambda_{+}^{2}\left(P_{A}\right)^{\mu \nu}{ }_{\nu^{\prime} \rho^{\prime \prime}}\left(P_{A}\right)_{\rho^{\prime} \sigma^{\prime}}^{\rho \sigma} g^{\rho^{\prime \prime} \rho^{\prime}} L^{\nu^{\prime} \sigma^{\prime}}
$$

where $P_{A}$ and $g_{\mu \nu}$ respectively stand for an antisymmetrizer and the quantum metric related to $U_{q}(s o(4))$.

The quantum algebra $U_{q}(s o(4))$ has two Casimir operators. They are

$$
\begin{aligned}
C_{1} & \equiv g_{\mu \nu} g_{\rho \sigma} L^{\mu \rho} L^{\nu \sigma} \\
& =2 L^{23} L^{23}+\lambda_{+}\left(L^{12} L^{34}+L^{13} L^{24}\right)
\end{aligned}
$$




$$
\begin{aligned}
& +q^{2} \lambda_{+}\left(L^{24} L^{13}+L^{34} L^{12}\right)+\left(q^{2}+q^{-2}\right) L^{14} L^{14} \\
& -\lambda\left(L^{14} L^{23}+L^{23} L^{14}\right)
\end{aligned}
$$

and

$$
\begin{aligned}
C_{2} \equiv & \varepsilon_{\mu \nu \rho \sigma} L^{\mu \nu} L^{\rho \sigma} \\
= & q^{2} \lambda_{+}^{2}\left(L^{14} L^{23}+L^{23} L^{14}\right)+q^{2} \lambda_{+}^{2}\left(L^{12} L^{34}-L^{13} L^{24}\right) \\
& +q^{4} \lambda_{+}^{2}\left(L^{34} L^{12}-L^{24} L^{13}\right)-q^{2} \lambda \lambda_{+}^{2} L^{14} L^{14},
\end{aligned}
$$

where $\varepsilon_{\mu \nu \rho \sigma}$ denotes the totally antisymmetric tensor of four-dimensional $q$ deformed Euclidean space.

The Euclidean algebra of four-dimensional $q$-deformed Euclidean space is again a cross product of the quantum algebra $U_{q}(s o(4))$ and the momentum algebra subject to the relations

$$
\begin{gathered}
P^{1} P^{\mu}=q P^{\mu} P^{1}, \quad P^{\mu} P^{4}=q P^{\mu} P^{3}, \quad \mu=2,3 \\
P^{2} P^{3}=P^{3} P^{2}, \quad P^{4} P^{1}=P^{1} P^{4}+\lambda P^{2} P^{3} .
\end{gathered}
$$

For the sake of completeness let us note that the above relations can alternatively be formulated by means of the antisymmetrizer $P_{A}$ of four-dimensional q-deformed Euclidean space:

$$
\left(P_{A}\right)_{\mu^{\prime} \nu^{\prime}}^{\mu \nu} P^{\mu^{\prime}} P^{\nu^{\prime}}=0 .
$$

The momentum components $P^{\mu}, \mu=1, \ldots, 4$, transform under $U_{q}(\operatorname{so}(4))$ as a vector operator. The commutation relations between the components of the antisymmetric tensor operator $L^{\mu \nu}$ and the momentum components $P^{\mu}$ were presented in Ref. [60]. The corresponding $q$-commutators take the general form

$$
\left[L^{\mu \nu}, P^{\rho}\right]_{q}=-q^{-1} \lambda_{+}\left(P_{A}\right)^{\mu \nu}{ }_{\nu^{\prime} \rho^{\prime}} g^{\rho^{\prime} \rho} P^{\nu^{\prime}}
$$

The four-dimensional $q$-deformed Euclidean algebra has two Casimir operators. It should be clear that one Casimir is given by the mass square

$$
m^{2} \equiv g_{\mu \nu} P^{\mu} P^{\nu}
$$

To get the second Casimir operator we are looking for a right-vector operator whose components $W^{\mu}, \mu=1, \ldots, 4$, commute with momenta. Making suitable ansaetze for the $W^{\mu}, \mu=1, \ldots, 4$, within the Euclidean algebra we are finally led to

$$
W^{1}=2 \lambda^{-1}\left(K_{1}^{1 / 2} K_{2}^{-1 / 2}-K_{1}^{-1 / 2} K_{2}^{1 / 2}\right) P^{1}
$$




$$
\begin{aligned}
& +2 q^{-1}\left(K_{1}^{1 / 2} K_{2}^{-1 / 2} P^{2} L_{1}^{-}-K_{1}^{-1 / 2} K_{2}^{1 / 2} P^{3} L_{2}^{-}\right) \\
W^{2}= & 2 \lambda^{-1}\left(K_{1}^{1 / 2} K_{2}^{1 / 2}+K_{1}^{-1 / 2} K_{2}^{-1 / 2}\right) P^{2} \\
& +2 q K_{1}^{1 / 2} K_{2}^{1 / 2} P^{1} L_{1}^{+}+2 q^{-1} K_{1}^{1 / 2} K_{2}^{1 / 2} P^{4} L_{2}^{-} \\
& +2 \lambda K_{1}^{1 / 2} K_{2}^{1 / 2} P^{3} L_{2}^{-} L_{1}^{+}, \\
W^{3}= & 2 \lambda^{-1}\left(K_{1}^{1 / 2} K_{2}^{1 / 2}+K_{1}^{-1 / 2} K_{2}^{-1 / 2}\right) P^{3} \\
& -2 q K_{1}^{1 / 2} K_{2}^{1 / 2} P^{1} L_{2}^{+}-2 q^{-1} K_{1}^{1 / 2} K_{2}^{1 / 2} P^{4} L_{1}^{-} \\
& -2 \lambda K_{1}^{1 / 2} K_{2}^{1 / 2} P^{2} L_{1}^{-} L_{2}^{+}, \\
W^{4}= & 2 \lambda^{-1}\left(K_{1}^{-1 / 2} K_{2}^{1 / 2}+K_{1}^{1 / 2} K_{2}^{-1 / 2}\right) P^{4} \\
& +2 q\left(K_{1}^{-1 / 2} K_{2}^{1 / 2} P^{2} L_{2}^{+}-K_{1}^{1 / 2} K_{2}^{-1 / 2} P^{3} L_{1}^{+}\right) .
\end{aligned}
$$

Notice that in the undeformed limit these components pass into the classical expressions $W^{\mu}=\varepsilon^{\mu \nu \rho \sigma} P_{\nu} L_{\rho \sigma}$. From the $W^{\mu}$ we can build a non-trivial Casimir operator via

$$
W^{2} \equiv g_{\mu \nu} W^{\nu} W^{\mu}
$$

Written out it reads

$$
\begin{aligned}
8^{-1} W^{2}= & \lambda^{-2} \lambda_{+}^{-1}\left(P^{1} P^{4}+q P^{2} P^{3}\right) \\
& -\lambda^{-2} P^{2} P^{3}\left(K_{1} K_{2}+q^{2} K_{1}^{-1} K_{2}^{-1}\right) \\
& +q \lambda^{-2} P^{1} P^{4}\left(K_{1}^{-1} K_{2}+K_{1} K_{2}^{-1}+\lambda K_{1} K_{2}\right) \\
& +\lambda^{-1} P^{1} P^{3} K_{1} K_{2} L_{1}^{+}+\lambda^{-1} P^{1} P^{2} K_{1} K_{2} L_{2}^{+} \\
& +q \lambda^{-1}\left(P^{2} P^{4} K_{1}\left(K_{2}-K_{2}^{-1}\right) L_{1}^{-}+P^{3} P^{4}\left(K_{1}-K_{1}^{-1}\right) K_{2} L_{2}^{-}\right) \\
& -\left(P^{2} P^{3} K_{1} K_{2}^{-1}+q^{-1} P^{1} P^{4} K_{1} K_{2}\right) L_{1}^{+} L_{1}^{-} \\
& -\left(P^{2} P^{3} K_{1}^{-1} K_{2}+q^{-1} P^{1} P^{4} K_{1} K_{2}\right) L_{2}^{+} L_{2}^{-} \\
& -q^{-1}\left(P^{1}\right)^{2} K_{1} K_{2} L_{1}^{+} L_{2}^{+}+\left(P^{2}\right)^{2} K_{1} K_{2} L_{1}^{-} L_{2}^{+} \\
& +\left(P^{3}\right)^{2} K_{1} K_{2} L_{1}^{+} L_{2}^{-}-q\left(P^{4}\right)^{2} K_{1} K_{2} L_{1}^{-} L_{2}^{-} \\
& -q^{-2} \lambda\left(P^{1} P^{2} K_{1} K_{2} L_{1}^{+} L_{1}^{-} L_{2}^{+}+P^{1} P^{3} K_{1} K_{2} L_{1}^{+} L_{2}^{+} L_{2}^{-}\right) \\
& -q^{-1} \lambda\left(P^{2} P^{4} K_{1} K_{2} L_{1}^{-} L_{2}^{+} L_{2}^{-}+P^{3} P^{4} K_{1} K_{2} L_{1}^{+} L_{1}^{-} L_{2}^{-}\right) \\
& -q^{-2} \lambda P^{2} P^{3} K_{1} K_{2} L_{1}^{+} L_{1}^{-} L_{2}^{+} L_{2}^{-} .
\end{aligned}
$$


This operator commutes with all elements of $U_{q}(s o(4))$, since it is defined as square of a right-vector. The fact that the components $W^{\mu}$ commute with momenta implies the same for $W^{2}$.

\subsection{Symmetry algebra in spinor notation}

It is sometimes helpful to introduce a new set of generators for $U_{q}(s o(4))$, replacing the generators $L^{\mu \nu}$ with vector indices by the generators $M^{\alpha \beta}$ and $\tilde{M}^{\dot{\alpha} \dot{\beta}}$ with spinor indices:

$$
\begin{gathered}
M^{\alpha \beta}=k_{1}\left(\sigma_{\mu \nu}^{-1}\right)^{\alpha \beta} L^{\mu \nu}, \\
\tilde{M}^{\dot{\alpha} \dot{\beta}}=k_{2}\left(\tilde{\sigma}_{\mu \nu}^{-1}\right)^{\dot{\alpha} \dot{\beta}} L^{\mu \nu}, \\
L^{\mu \nu}=k_{1}^{\prime}\left(\sigma^{\mu \nu}\right)_{\alpha \beta} M^{\alpha \beta}+k_{2}^{\prime}\left(\tilde{\sigma}^{\mu \nu}\right)_{\dot{\alpha} \dot{\beta}} \tilde{M}^{\dot{\alpha} \dot{\beta}},
\end{gathered}
$$

where the constants have to satisfy the condition

$$
k_{1} k_{1}^{\prime}=k_{2} k_{2}^{\prime}=\lambda_{+}^{-1} .
$$

More explicitly, we have

$$
\begin{gathered}
M^{11}=-k_{1} q^{1 / 2} \lambda_{+} L^{13}, \quad M^{22}=k_{1} q^{1 / 2} \lambda_{+} L^{24}, \\
M^{12}=q M^{21}=k_{1} q^{1 / 2}\left(q L^{14}-L^{23}\right), \\
\tilde{M}^{11}=-k_{2} q^{1 / 2} \lambda_{+} L^{12}, \quad \tilde{M}^{22}=k_{2} q^{1 / 2} \lambda_{+} L^{34}, \\
\tilde{M}^{12}=q \tilde{M}^{21}=k_{2} q^{-1 / 2} L^{14}+k_{2} q^{1 / 2} L^{23},
\end{gathered}
$$

and

$$
\begin{gathered}
L^{12}=k_{2}^{\prime} q^{-1 / 2} \tilde{M}^{11}, \quad L^{13}=k_{1}^{\prime} q^{-1 / 2} M^{11}, \\
L^{14}=-k_{2}^{\prime} q^{-1 / 2} \tilde{M}^{12}-k_{1}^{\prime} q^{-1 / 2} M^{12}, \\
L^{23}=-k_{2}^{\prime} q^{1 / 2} \tilde{M}^{12}+k_{1}^{\prime} q^{-3 / 2} M^{12}, \\
L^{24}=k_{1}^{\prime} q^{-1 / 2} M^{22}, \quad L^{34}=-k_{2}^{\prime} q^{-1 / 2} \tilde{M}^{22} .
\end{gathered}
$$

The $q$-commutators of the quantum Lie algebra of $U_{q}(s o(4))$ in terms of $M^{\alpha \beta}$ and $\tilde{M}^{\dot{\alpha} \dot{\beta}}$ are given by

$$
\left[M^{\alpha \beta}, M^{\gamma \delta}\right]_{q}=\left(k_{1}^{\prime}\right)^{-1} q^{-1} \lambda_{+} S_{\beta^{\prime} \alpha^{\prime}}^{\alpha \beta}{ }_{\delta^{\prime} \gamma^{\prime}} \varepsilon^{\alpha^{\prime} \delta^{\prime}} M^{\beta^{\prime} \gamma^{\prime}},
$$




$$
\begin{gathered}
{\left[\tilde{M}^{\dot{\alpha} \dot{\beta}}, \tilde{M}^{\dot{\gamma} \dot{\delta}}\right]_{q}=\left(k_{2}^{\prime}\right)^{-1} q^{-1} \lambda_{+} S^{\dot{\alpha} \dot{\beta}}{ }_{\dot{\beta}^{\prime} \dot{\alpha}^{\prime}} S_{\dot{\gamma}^{\prime} \dot{\gamma}^{\prime} \dot{\gamma}^{\prime}} \varepsilon^{\dot{\alpha}^{\prime} \dot{\delta}^{\prime}} \tilde{M}^{\dot{\beta}^{\prime} \dot{\gamma}^{\prime}},} \\
{\left[M^{\alpha \beta}, \tilde{M}^{\dot{\gamma} \dot{\delta}}\right]_{q}=\left[\tilde{M}^{\dot{\alpha} \dot{\beta}}, M^{\gamma \delta}\right]_{q}=0 .}
\end{gathered}
$$

In spinor notation the two Casimirs of $U_{q}(s o(4))$ [cf. Eqs. (69) and (70)] become

$$
\begin{aligned}
C_{1}= & -k_{1}^{\prime 2} \lambda_{+} \varepsilon_{\alpha \beta} \varepsilon_{\alpha^{\prime} \beta^{\prime}} M^{\alpha \alpha^{\prime}} M^{\beta \beta^{\prime}} \\
& -k_{2}^{\prime 2} \lambda_{+} \varepsilon_{\dot{\alpha} \dot{\beta}} \varepsilon_{\dot{\alpha}^{\prime} \dot{\beta}^{\prime}} \tilde{M}^{\dot{\alpha} \dot{\alpha}^{\prime}} \tilde{M}^{\dot{\beta} \dot{\beta}^{\prime}}, \\
C_{2}= & k_{1}^{\prime 2} q^{2} \lambda_{+}^{2} \varepsilon_{\alpha \beta} \varepsilon_{\alpha^{\prime} \beta^{\prime}} M^{\alpha \alpha^{\prime}} M^{\beta \beta^{\prime}} \\
& -k_{2}^{\prime 2} q^{2} \lambda_{+}^{2} \varepsilon_{\dot{\alpha} \dot{\beta}^{\prime}} \varepsilon_{\dot{\alpha}^{\prime} \dot{\beta}^{\prime}} \tilde{M}^{\dot{\alpha} \dot{\alpha}^{\prime}} \tilde{M}^{\dot{\beta} \dot{\beta}^{\prime}} .
\end{aligned}
$$

These expressions are linked to the Casimir operators of the two $U_{q}(s u(2))$ subalgebras of $U_{q}(s o(4))$ :

$$
\begin{aligned}
& C_{1}+C_{2}=-k_{2}^{\prime 2} \lambda_{+}\left(1+q^{2} \lambda_{+}\right) \varepsilon_{\dot{\alpha} \dot{\beta}} \varepsilon_{\dot{\alpha}^{\prime} \dot{\beta}^{\prime}} \tilde{M}^{\dot{\alpha} \dot{\alpha}^{\prime}} \tilde{M}^{\dot{\beta} \dot{\beta}^{\prime}}, \\
& C_{1}-C_{2}=-k_{1}^{\prime 2} \lambda_{+}\left(1+q^{2} \lambda_{+}\right) \varepsilon_{\alpha \beta} \varepsilon_{\alpha^{\prime} \beta^{\prime}} M^{\alpha \alpha^{\prime}} M^{\beta \beta^{\prime}} .
\end{aligned}
$$

\subsection{The four-dimensional $q$-deformed Euclidean super- algebra}

In analogy to the three-dimensional case the four-dimensional $q$-deformed Euclidean superalgebra is generated by the generators of $U_{q}(s o(4))$, the momentum $P^{\mu}$, and the supersymmetry generators $Q^{\alpha}, \tilde{Q}^{\dot{\alpha}}, \alpha, \dot{\alpha}=1,2$. Again, the supersymmetry generators carry spinor indices. Thus, the $Q^{\alpha}$ as well as the $\tilde{Q}^{\dot{\alpha}}$ span an antisymmetrized quantum plane, i.e.

$$
\begin{gathered}
Q^{\alpha} Q^{\alpha}=\tilde{Q}^{\dot{\alpha}} \tilde{Q}^{\dot{\alpha}}=0, \quad \alpha, \dot{\alpha}=1,2, \\
Q^{1} Q^{2}=-q^{-1} Q^{2} Q^{1}, \quad \tilde{Q}^{1} \tilde{Q}^{2}=-q^{-1} \tilde{Q}^{2} \tilde{Q}^{1} .
\end{gathered}
$$

Furthermore, the supersymmetry generators transform as spinor operators under $U_{q}(s o(4))$. More concretely, they refer to the representations $(1 / 2,0)$ and $(0,1 / 2)$. On these grounds, the $q$-commutators between the supersymmetry generators and the $L^{\mu \nu}$ become

$$
\begin{aligned}
& {\left[L^{\mu \nu}, Q^{\alpha}\right]_{q}=-q^{-1}\left(\sigma^{\mu \nu}\right)_{\beta}{ }^{\alpha} Q^{\beta},} \\
& {\left[L^{\mu \nu}, \tilde{Q}^{\dot{\alpha}}\right]_{q}=-q^{-1}\left(\tilde{\sigma}^{\mu \nu}\right)_{\dot{\beta}}{ }^{\dot{\alpha}} \tilde{Q}^{\dot{\beta}},}
\end{aligned}
$$


where $\sigma^{\mu \nu}$ and $\tilde{\sigma}^{\mu \nu}$ denote the two-dimensional spin matrices to four-dimensional $q$-deformed Euclidean space (see Ref. [59]). For the explicit form of the above $q$-commutators we refer the reader to Ref. [60]. In spinor notation we have

$$
\begin{gathered}
{\left[M^{\alpha \beta}, Q^{\gamma}\right]_{q}=k_{1}^{-1} q^{-1} S_{\alpha^{\prime} \beta^{\prime}}^{\alpha \beta} \varepsilon^{\beta^{\prime} \gamma} Q^{\alpha^{\prime}},} \\
{\left[\tilde{M}^{\dot{\alpha} \dot{\beta}}, \tilde{Q}^{\dot{\gamma}}\right]_{q}=k_{2}^{-1} q^{-1} S_{\dot{\alpha}^{\prime} \dot{\beta}^{\prime}}^{\dot{\alpha} \dot{\beta}} \varepsilon^{\dot{\beta}^{\prime} \dot{\gamma}} \tilde{Q}^{\dot{\alpha}^{\prime}},} \\
{\left[M^{\alpha \beta}, \tilde{Q}^{\dot{\gamma}}\right]_{q}=\left[\tilde{M}^{\dot{\alpha} \dot{\beta}}, Q^{\gamma}\right]_{q}=0 .}
\end{gathered}
$$

Next, we come to the relations between the generators $Q^{\alpha}$ and $\tilde{Q}^{\dot{\alpha}}$. These relations are covariant with respect to $U_{q}(s o(4))$ if they take the form

$$
\begin{aligned}
& \tilde{Q}^{1} Q^{1}+Q^{1} \tilde{Q}^{1}=c P^{1}, \\
& \tilde{Q}^{1} Q^{2}+Q^{2} \tilde{Q}^{1}=c P^{2}, \\
& \tilde{Q}^{2} Q^{1}+Q^{1} \tilde{Q}^{2}=c P^{3}, \\
& \tilde{Q}^{2} Q^{2}+Q^{2} \tilde{Q}^{2}=-c P^{4},
\end{aligned}
$$

where $c$ again denotes an undetermined constant, which we can set equal to 1. With the help of the Pauli matrices for $q$-deformed Euclidean space in four dimensions (see Ref. [59] ) the above relations can be written as

$$
\left\{\tilde{Q}^{\dot{\alpha}}, Q^{\beta}\right\}=\left(\tilde{\sigma}_{\mu}^{-1}\right)^{\alpha \dot{\beta}} P^{\mu} .
$$

Notice that the anticommutator in the last formula is the ordinary one.

Last but not least, we have to specify how the momentum operators commute with the supersymmetry generators. Again, the commutation relations between momentum operators and supersymmetry generators are uniquely determined by the requirement that they have to be consistent with our previous relations. This way, we have

$$
\begin{aligned}
& P^{1} Q^{1}=Q^{1} P^{1}, \quad P^{2} Q^{2}=Q^{2} P^{2}, \\
& P^{3} Q^{1}=Q^{1} P^{3}, \quad P^{4} Q^{2}=Q^{2} P^{4}, \\
& P^{2} Q^{1}=q^{-1} Q^{1} P^{2}, \\
& P^{4} Q^{1}=q^{-1} Q^{1} P^{4}, \\
& P^{1} Q^{2}=q^{-1} Q^{2} P^{1}+q^{-1} \lambda Q^{1} P^{2}, \\
& P^{3} Q^{2}=q^{-1} Q^{2} P^{3}-q^{-1} \lambda Q^{1} P^{4},
\end{aligned}
$$


and

$$
\begin{aligned}
& P^{1} \tilde{Q}^{1}=\tilde{Q}^{1} P^{1}, \quad P^{2} \tilde{Q}^{1}=\tilde{Q}^{1} P^{2}, \\
& P^{3} \tilde{Q}^{2}=\tilde{Q}^{2} P^{3}, \quad P^{4} \tilde{Q}^{2}=\tilde{Q}^{2} P^{4}, \\
& P^{1} \tilde{Q}^{2}=q \tilde{Q}^{2} P^{1}, \\
& P^{2} \tilde{Q}^{2}=q \tilde{Q}^{2} P^{2}, \\
& P^{3} \tilde{Q}^{1}=q \tilde{Q}^{1} P^{3}-q \lambda \tilde{Q}^{2} P^{1}, \\
& P^{4} \tilde{Q}^{1}=q \tilde{Q}^{1} P^{4}+q \lambda \tilde{Q}^{2} P^{2} .
\end{aligned}
$$

These relations can again be generated from $q$-commutators:

$$
\left[P^{\mu}, Q^{\alpha}\right]_{\bar{q}}=0, \quad\left[P^{\mu}, \tilde{Q}^{\dot{\alpha}}\right]_{q}=0 .
$$

Notice that the two types of $q$-commutators are defined as in (60), but now we have to use the Hopf structures to four-dimensional $q$-deformed Euclidean space (see Ref. [32,35]). As usual these Hopf structures depend on a unitary scaling operator with

$$
\Lambda Q^{\alpha}=q Q^{\alpha} \Lambda, \quad \Lambda \tilde{Q}^{\dot{\alpha}}=q \tilde{Q}^{\dot{\alpha}} \Lambda .
$$

Now, we have everything together to write down the q-deformed Euclidean superalgebra in four dimensions:

$$
\begin{gathered}
{\left[L^{\mu \nu}, L^{\rho \sigma}\right]_{q}=-q^{-1} \lambda_{+}^{2}\left(P_{A}\right)_{{ }^{\prime} \rho^{\prime \prime}}^{\mu \nu}\left(P_{A}\right)_{\rho^{\prime} \sigma^{\prime}}^{\rho \sigma} g^{\rho^{\prime \prime} \rho^{\prime}} L^{\nu^{\prime} \sigma^{\prime}}} \\
{\left[L^{\mu \nu}, P^{\rho}\right]_{q}=-q^{-1} \lambda_{+}\left(P_{A}\right)^{\mu \nu}{ }_{\nu^{\prime} \rho^{\prime}} g^{\rho^{\prime} \rho} P^{\nu^{\prime}}} \\
{\left[L^{\mu \nu}, Q^{\alpha}\right]_{q}=-q^{-1}\left(\sigma^{\mu \nu}\right)_{\beta}{ }^{\alpha} Q^{\beta}} \\
{\left[L^{\mu \nu}, \tilde{Q}^{\dot{\alpha}}\right]_{q}=-q^{-1}\left(\tilde{\sigma}^{\mu \nu}\right)_{\dot{\beta}}{ }^{\dot{\alpha}} \tilde{Q}^{\dot{\beta}}} \\
\left(P_{A}\right)^{\mu \nu}{ }_{\mu^{\prime} \nu^{\prime}} P^{\mu^{\prime}} P^{\nu^{\prime}}=0 \\
{\left[P^{\mu}, Q^{\alpha}\right]_{\bar{q}}=0, \quad\left[P^{\mu}, \tilde{Q}^{\dot{\alpha}}\right]_{q}=0} \\
\left\{Q^{\alpha}, Q^{\beta}\right\}_{q}=0, \quad\left\{\tilde{Q}^{\dot{\alpha}}, \tilde{Q}^{\dot{\beta}}\right\}_{q}=0 \\
\left\{\tilde{Q}^{\dot{\alpha}}, Q^{\beta}\right\}=\left(\tilde{\sigma}_{\mu}^{-1}\right)^{\dot{\alpha} \beta} P^{\mu} .
\end{gathered}
$$

It should be remarked that conjugating these relations and making use of the conjugation properties

$$
\overline{L^{\mu \nu}}=g_{\mu \mu^{\prime}} g_{\nu \nu^{\prime}} L^{\nu^{\prime} \mu^{\prime}}, \quad \overline{P^{\mu}}=g_{\mu \mu^{\prime}} P^{\mu^{\prime}},
$$




$$
\overline{Q^{\alpha}}=\varepsilon_{\alpha \alpha^{\prime}} Q^{\alpha^{\prime}}, \quad \overline{\tilde{Q}^{\alpha}}=-\varepsilon_{\alpha \alpha^{\prime}} \tilde{Q}^{\alpha^{\prime}},
$$

we obtain a second four-dimensional $q$-deformed Euclidean superalgebra, which differs from the above one by the commutation relations between momentum generators and supergenerators, only. Instead of (96) and (97) we would have

$$
\begin{aligned}
& P^{1} Q^{1}=Q^{1} P^{1}, \quad P^{2} Q^{2}=Q^{2} P^{2}, \\
& P^{3} Q^{1}=Q^{1} P^{3}, \quad P^{4} Q^{2}=Q^{2} P^{4}, \\
& P^{1} Q^{2}=q Q^{2} P^{1}, \quad P^{3} Q^{2}=q Q^{2} P^{3}, \\
& P^{2} Q^{1}=q Q^{1} P^{2}-q \lambda Q^{2} P^{1} \\
& P^{4} Q^{1}=q Q^{1} P^{4}+q \lambda Q^{2} P^{3} .
\end{aligned}
$$

and

$$
\begin{aligned}
& P^{1} \tilde{Q}^{1}=\tilde{Q}^{1} P^{1}, \quad P^{2} \tilde{Q}^{1}=\tilde{Q}^{1} P^{2}, \\
& P^{3} \tilde{Q}^{2}=\tilde{Q}^{2} P^{3}, \quad P^{4} \tilde{Q}^{2}=\tilde{Q}^{2} P^{4} \\
& P^{3} \tilde{Q}^{1}=q^{-1} \tilde{Q}^{1} P^{3}, \quad P^{4} \tilde{Q}^{1}=q^{-1} \tilde{Q}^{1} P^{4} \\
& P^{1} \tilde{Q}^{2}=q^{-1} \tilde{Q}^{2} P^{1}+q^{-1} \lambda \tilde{Q}^{1} P^{3} \\
& P^{2} \tilde{Q}^{2}=q^{-1} \tilde{Q}^{2} P^{2}-q^{-1} \lambda \tilde{Q}^{1} P^{4} .
\end{aligned}
$$

In terms of $q$-commutators these relations become

$$
\left[P^{\mu}, Q^{\alpha}\right]_{q}=0, \quad\left[P^{\mu}, \tilde{Q}^{\dot{\alpha}}\right]_{\bar{q}}=0 .
$$

\section{Symmetry algebras for $q$-deformed Minkow- ski space}

In this section we apply our considerations to $q$-deformed Minkowski space leading us to a $q$-analog of the Poincaré superalgebra. From a physical point of view this case is the most interesting one.

\subsection{The $q$-deformed Poincaré algebra}

The $q$-deformed Poincaré algebra is a cross product of the $q$-deformed Lorentz algebra and the momentum algebra of $q$-deformed Minkowski space. In our 
work we often use a formulation of $q$-deformed Lorentz algebra as it was given in Ref. [46]. In Ref. [60], however, we considered as Lorentz generators the components of an antisymmetric tensor of second rank. Its components $V^{\mu \nu}, \mu, \nu \in\{0,+,-, 3\}$, enable us to formulate the quantum Lie algebra of the $q$-deformed Lorentz algebra as follows:

$$
\left[V^{\mu \nu}, V^{\rho \sigma}\right]_{q}=-q^{-1} \lambda_{+}\left(P_{A}\right)^{\mu \nu}{ }_{\nu^{\prime} \rho^{\prime \prime}}\left(P_{A}\right)^{\rho \sigma}{ }_{\rho^{\prime} \sigma^{\prime}} \eta^{\rho^{\prime \prime} \rho^{\prime}} V^{\nu^{\prime} \sigma^{\prime}},
$$

where $\eta_{\mu \nu}$ and $P_{A}$ respectively stand for the quantum metric and an antisymmetrizer of $q$-deformed Minkowski. For the explicit form of these $q$ commutators we again refer to Ref. [60]. From the same reference we know the two Casimirs of $q$-deformed Lorentz algebra:

$$
C_{1} \equiv \eta_{\mu \nu} \eta_{\rho \sigma} V^{\mu \rho} V^{\nu \sigma}, \quad C_{2} \equiv \varepsilon_{\mu \nu \rho \sigma} V^{\mu \nu} V^{\rho \sigma} .
$$

The momentum algebra of $q$-deformed Minkowski space is spanned by the momentum components $P^{\mu}, \mu \in\{0,+,-, 3\}$, subject to

$$
\begin{gathered}
P^{\mu} P^{0}=P^{0} P^{\mu}, \quad \mu \in\{0,+,-, 3\} \\
P^{3} P^{ \pm}-q^{ \pm 2} P^{ \pm} P^{3}=-q \lambda P^{0} P^{ \pm}, \\
P^{-} P^{+}-P^{+} P^{-}=\lambda\left(P^{3} P^{3}-P^{0} P^{3}\right) .
\end{gathered}
$$

Let us recall that the $P^{\mu}, \mu \in\{0,+,-, 3\}$, behave as a four-vector operator under $q$-deformed Lorentz transformations. For this reason, the commutation relations between generators of the $q$-deformed Lorentz algebra and the corresponding momentum components take the form (see also Ref. [60])

$$
\left[V^{\mu \nu}, P^{\rho}\right]_{q}=-q^{-1}\left(P_{A}\right)^{\mu \nu}{ }_{\nu^{\prime} \rho^{\prime}} \eta^{\rho^{\prime} \rho} P^{\nu^{\prime}} .
$$

The Casimir operators of $q$-deformed Poincaré algebra are found from the same reasonings already applied to the four-dimensional $q$-deformed Euclidean algebra. In this manner, one Casimir is given by the mass square

$$
m^{2} \equiv g_{\mu \nu} P^{\mu} P^{\nu},
$$

and the $q$-analog of the spin Casimir becomes $[69,70]$

$$
W^{2} \equiv \eta_{\nu \mu} W^{\mu} W^{\nu}
$$

where we have to take as components of the $q$-deformed Pauli-Lubanskivector:

$$
W^{+}=q^{2} \lambda^{-1} P^{+}-q^{2} \lambda^{-1} P^{+}\left(\tau^{3}\right)^{1 / 2}\left(\tau^{1}\right)^{2}-q^{5} \lambda P^{-}\left(\tau^{3}\right)^{1 / 2}\left(T^{2}\right)^{2}
$$




$$
\begin{aligned}
& -q^{9 / 2} \lambda_{+}^{1 / 2}\left(\tau^{3}\right)^{1 / 2} P^{3} T^{2} \tau^{1}+q^{5 / 2} \lambda_{+}^{-1 / 2}\left(P^{3}-P^{0}\right) T^{+} \\
W^{3}= & -\lambda^{-1} P^{3}-q^{-1 / 2} \lambda_{+}^{1 / 2} P^{-} T^{2} \sigma^{2}-q^{1 / 2} \lambda_{+}^{1 / 2} P^{+} S^{1} \tau^{1} \\
& -q \lambda \lambda_{+} P^{3} T^{2} S^{1}-q \lambda \lambda_{+}^{-1}\left(P^{3}-P^{0}\right)\left(\tau^{3}\right)^{1 / 2} T^{+} T^{-} \\
& +q^{-1 / 2} \lambda_{+}^{-1 / 2} P^{-}\left(\tau^{3}\right)^{-1 / 2} T^{+}-q^{1 / 2} \lambda_{+}^{-1 / 2} P^{+}\left(\tau^{3}\right)^{-1 / 2} T^{-} \\
& +\lambda^{-1} \lambda_{+}^{-1}\left(\left(q^{-1} P^{3}-q^{-1} P^{0}\right)\left(\tau^{3}\right)^{1 / 2}+\left(q P^{3}+q^{-1} P^{0}\right)\left(\tau^{3}\right)^{-1 / 2}\right), \\
W^{0}= & -\lambda^{-1} P^{0}+q \lambda \lambda_{+}^{-1}\left(P^{0}-P^{3}\right)\left(\tau^{3}\right)^{-1 / 2} T^{+} T^{-} \\
& +\lambda^{-1} \lambda_{+}^{-1}\left(q\left(P^{0}-P^{3}\right)\left(\tau^{3}\right)^{1 / 2}+\left(q^{-1} P^{0}+q P^{3}\right)\left(\tau^{3}\right)^{-1 / 2}\right) \\
& +q^{-1 / 2} \lambda_{+}^{-1 / 2} P^{-}\left(\tau^{3}\right)^{-1 / 2} T^{+}-q^{1 / 2} \lambda_{+}^{-1 / 2} P^{+}\left(\tau^{3}\right)^{-1 / 2} T^{-} \\
W^{-}= & q^{2} \lambda^{-1} P^{-}-q^{-5 / 2} \lambda_{+}^{-1 / 2}\left(P^{3}-P^{0}\right) T^{-}-q \lambda P^{+}\left(\tau^{3}\right)^{-1 / 2}\left(S^{1}\right)^{2} \\
& -q^{-1 / 2} \lambda_{+}^{1 / 2} P^{3}\left(\tau^{3}\right)^{-1 / 2} S^{1} \sigma^{2}+q^{-2} \lambda^{-1} P^{-}\left(\tau^{3}\right)^{-1 / 2}\left(\sigma^{2}\right)^{2} .
\end{aligned}
$$

where $S^{1}, T^{2}, \tau^{1}, \sigma^{2}$ denote generators of the $q$-deformed Lorentz algebra [46]. Written out explicitly, the $q$-deformed spin Casimir reads

$$
\begin{aligned}
2^{-1} W^{2}= & \lambda^{-2}\left(q^{-2}\left(P^{3}\right)^{2}-\left(P^{0}\right)^{2}+q^{-1} \lambda P^{3} P^{0}-\lambda_{+} P^{+} P^{-}\right) \\
& -\left(P^{+}\right)^{2}\left(\tau^{3}\right)^{-1 / 2}\left[q T^{-} S^{1} \tau^{1}-q^{2}\left(S^{1}\right)^{2}\right] \\
& -\lambda_{+}^{-1}\left(P^{0}\right)^{2}\left(\tau^{3}\right)^{-1 / 2}\left[q^{-1} T^{+} T^{-}+\lambda^{-2}\left(q \tau^{3}+q^{-1} \mathbb{1}\right)\right] \\
& +\left(P^{-}\right)^{2}\left(\tau^{3}\right)^{-1 / 2}\left[q^{-1} T^{+} T^{2} \sigma^{2}-q^{4} \tau^{3}\left(T^{2}\right)^{2}\right] \\
& +\left(P^{3}\right)^{2}\left(\tau^{3}\right)^{-1 / 2}\left[q \tau^{3} T^{-} T^{2} \tau^{1}-q^{-1} T^{+} S^{1} \sigma^{2}\right. \\
& -q^{-1} \lambda^{-1}\left(\sigma^{2}\right)^{2}-\lambda T^{-} T^{2} \sigma^{2}+\left(\tau^{3}+q^{2} \mathbb{1}-\lambda^{2} T^{+} T^{-}\right) T^{2} S^{1} \\
& \left.+\lambda^{-2} \lambda_{+}^{-1}\left(q \mathbb{1}+q^{-1} \tau^{3}-q^{-1} \lambda^{2} T^{+} T^{-}\right)\right] \\
& +q^{-3 / 2} \lambda^{-1} \lambda_{+}^{-1 / 2}\left(P^{+} P^{3}-P^{+} P^{0}\right)\left(\tau^{3}\right)^{-1 / 2}\left[\tau^{3} T^{-}\left(\tau^{1}\right)^{2}\right. \\
& \left.+q \tau^{3} S^{1} \tau^{1}-q^{4} T^{-}-q \lambda^{2} T^{+} T^{-} S^{1} \tau^{1}-q^{2} \lambda^{2} T^{+}\left(S^{1}\right)^{2}\right] \\
& +q^{3 / 2} \lambda^{-1} \lambda_{+}^{-1 / 2} P^{+} P^{3}\left(\tau^{3}\right)^{-1 / 2}\left[S^{1} \tau^{1}-q S^{1} \sigma^{2}-q^{2} \lambda^{2} T^{-} T^{2} S^{1}\right] \\
& +q^{3 / 2} \lambda_{+}^{-1 / 2} P^{+} P^{0}\left(\tau^{3}\right)^{-1 / 2}\left[\lambda_{+} S^{1} \sigma^{2}+q \lambda^{2} T^{-} T^{2} S^{1}\right] \\
& \left.+P^{+} P^{-}\left(\tau^{3}\right)^{-1 / 2}\left[T^{+} S^{1} \tau^{1}-T^{-} T^{2} \sigma^{2}-\lambda^{-2}\left(q \tau^{3}\left(\tau^{1}\right)^{2}\right)+q^{-1}\left(\sigma^{2}\right)^{2}\right)\right] \\
& +P^{3} P^{0}\left(\tau^{3}\right)^{-1 / 2}\left[\left(\mathbb{1}-\tau^{3}\right) T^{2} S^{1}-\lambda \lambda_{+}^{-1}\left(\mathbb{1}-\lambda^{-2} \tau^{3}\right)\right. \\
& +q^{-1} T^{+} S^{1} \sigma^{2}-q \tau^{3} T^{-} T^{2} \tau^{1}+q^{-1} \lambda^{-1}\left(\sigma^{2}\right)^{2}
\end{aligned}
$$




$$
\begin{aligned}
& \left.+\lambda T^{-} T^{2} \sigma^{2}+\lambda^{2} T^{+} T^{-} T^{2} S^{1}\right] \\
& +q^{-1 / 2} \lambda_{+}^{-1 / 2}\left(P^{3} P^{-}-P^{0} P^{-}\right)\left(\tau^{3}\right)^{-1 / 2}\left[\lambda^{-1} T^{+}-\lambda^{-1} T^{+}\left(\sigma^{2}\right)^{2}\right. \\
& \left.+q \lambda^{-1} \tau^{3} T^{2} \sigma^{2}-q \lambda T^{+} T^{-} T^{2} \sigma^{2}+q^{4} \lambda \tau^{3} T^{-}\left(T^{2}\right)^{2}\right] \\
& +q^{1 / 2} \lambda_{+}^{1 / 2} P^{3} P^{-}\left(\tau^{3}\right)^{-1 / 2}\left[q^{3} \lambda^{-1} \lambda_{+}^{-1} T^{2} \sigma^{2}\right. \\
& \left.-\lambda T^{+} T^{2} S^{1}-q^{4} \lambda^{-1} \tau^{3} T^{2} \tau^{1}\right] \\
& -q^{1 / 2} \lambda^{-1} \lambda_{+}^{-1 / 2}\left(\lambda \lambda_{+}-1\right) P^{0} P^{-}\left(\tau^{3}\right)^{-1 / 2} T^{2} \sigma^{2} .
\end{aligned}
$$

\subsection{Symmetry algebra in spinor notation}

In this section we proceed in very much the same way as was done in Sec. 3.2 for the four-dimensional $q$-deformed Euclidean algebra. First, we introduce Lorentz generators with spinor indices

$$
\begin{gathered}
M^{\alpha \beta}=k_{1}\left(\sigma_{\mu \nu}^{-1}\right)^{\alpha \beta} V^{\mu \nu}, \\
\bar{M}^{\dot{\alpha} \dot{\beta}}=k_{2}\left(\bar{\sigma}_{\mu \nu}^{-1}\right)^{\dot{\alpha} \dot{\beta}} V^{\mu \nu}, \\
V^{\mu \nu}=k_{1}^{\prime}\left(\sigma^{\mu \nu}\right)_{\alpha \beta} M^{\alpha \beta}+k_{2}^{\prime}\left(\bar{\sigma}^{\mu \nu}\right)_{\dot{\alpha} \dot{\beta}} \bar{M}^{\dot{\alpha} \dot{\beta}},
\end{gathered}
$$

where the two-dimensional spin matrices of $q$-deformed Minkowski space can be found in Ref. [59]. For the above identities to be consistent with each other we have to require that the constants $k_{i}, k_{i}^{\prime}, i=1,2$, fulfill

$$
k_{1} k_{1}^{\prime}=k_{2} k_{2}^{\prime}=\lambda_{+}^{-1} \text {. }
$$

If we set $k_{1}=k_{2}$, the Lorentz generators $M^{\alpha \beta}$ and $\bar{M}^{\dot{\alpha} \dot{\beta}}$ show the conjugation properties

$$
\overline{M^{\alpha \beta}}=\varepsilon_{\dot{\alpha} \dot{\alpha}^{\prime}} \varepsilon_{\dot{\beta} \dot{\beta}^{\prime}} \bar{M}^{\dot{\beta}^{\prime} \dot{\alpha}^{\prime}} \quad \overline{\bar{M} \dot{\alpha} \dot{\beta}}=\varepsilon_{\alpha \alpha^{\prime}} \varepsilon_{\beta \beta^{\prime}} \bar{M}^{\beta^{\prime} \alpha^{\prime}} .
$$

More explicitly, we have

$$
\begin{aligned}
& M^{11}=k_{1} \lambda_{+}^{1 / 2}\left(V^{3-}-V^{0-}\right), \\
& M^{12}=q M^{21}=k_{1}\left(q^{-1 / 2} V^{+-}+q^{1 / 2} V^{30}\right), \\
& M^{22}=k_{1} \lambda_{+}^{1 / 2}\left(q^{-2} V^{+3}+V^{+0}\right) .
\end{aligned}
$$

and

$$
\bar{M}^{11}=-k_{2} \lambda_{+}^{1 / 2}\left(V^{0-}+q^{-2} V^{3-}\right),
$$




$$
\begin{aligned}
& \bar{M}^{12}=q \bar{M}^{21}=-k_{2} q^{1 / 2}\left(q V^{+-}-V^{30}\right), \\
& \bar{M}^{22}=-k_{2} \lambda_{+}^{1 / 2}\left(V^{+3}-V^{+0}\right) .
\end{aligned}
$$

Solving these equalities for the independent $V^{\mu \nu}$ yields

$$
\begin{aligned}
V^{+3} & =q \lambda_{+}^{-1 / 2}\left(k_{1}^{\prime} M^{22}-k_{2}^{\prime} \bar{M}^{22}\right), \\
V^{+0} & =\lambda_{+}^{-1 / 2}\left(k_{1}^{\prime} q M^{22}+k_{2}^{\prime} q^{-1} \bar{M}^{22}\right), \\
V^{+-} & =q^{-1 / 2}\left(k_{1}^{\prime} M^{12}-k_{2}^{\prime} \bar{M}^{12}\right), \\
V^{30} & =k_{1}^{\prime} q^{1 / 2} M^{12}+k_{2}^{\prime} q^{-3 / 2} \bar{M}^{12}, \\
V^{3-} & =q \lambda_{+}^{-1 / 2}\left(k_{1}^{\prime} M^{11}-k_{2}^{\prime} \bar{M}^{11}\right), \\
V^{0-} & =-\lambda_{+}^{-1 / 2}\left(k_{1}^{\prime} q^{-1} M^{11}+k_{2}^{\prime} q \bar{M}^{11}\right) .
\end{aligned}
$$

For the quantum Lie algebra in spinor notation we have

$$
\begin{aligned}
& {\left[M^{\alpha \beta}, M^{\gamma \delta}\right]_{q}=-\left(k_{1}^{\prime}\right)^{-1} S_{\beta^{\prime} \alpha^{\prime}}^{\alpha \beta} S_{\delta^{\prime} \gamma^{\prime}} \varepsilon^{\alpha^{\prime} \delta^{\prime}} M^{\beta^{\prime} \gamma^{\prime}},} \\
& {\left[\bar{M}^{\alpha \beta}, \bar{M}^{\dot{\gamma} \dot{\delta}}\right]_{q}=-\left(k_{2}^{\prime}\right)^{-1} S_{\dot{\beta}^{\prime} \dot{\alpha}^{\prime}}^{\dot{\alpha} \dot{\beta}} S_{\dot{\delta}^{\prime} \dot{\gamma}^{\prime}}^{\dot{\gamma} \dot{\alpha^{\prime}} \dot{\alpha}^{\prime} \dot{\delta}^{\prime}} \bar{M}^{\dot{\beta}^{\prime} \dot{\gamma}^{\prime}},} \\
& {\left[M^{\alpha \beta}, \bar{M}^{\dot{\gamma} \dot{\delta}}\right]_{q}=\left[\bar{M}^{\dot{\alpha} \dot{\beta}}, M^{\gamma \delta}\right]_{q}=0 .}
\end{aligned}
$$

In spinor notation the Casimirs of $q$-deformed Lorentz algebra become

$$
\begin{aligned}
C_{1}= & -k_{1}^{\prime 2} \lambda_{+} \varepsilon_{\alpha \beta} \varepsilon_{\alpha^{\prime} \beta^{\prime}} M^{\alpha \alpha^{\prime}} M^{\beta \beta^{\prime}} \\
& -k_{2}^{\prime 2} \lambda_{+} \varepsilon_{\dot{\alpha} \dot{\beta}} \varepsilon_{\dot{\alpha}^{\prime} \dot{\beta}^{\prime}} \bar{M}^{\dot{\alpha} \dot{\alpha}^{\prime}} \bar{M}^{\dot{\beta} \dot{\beta}^{\prime}}, \\
C_{2}= & -k_{1}^{\prime 2}\left(3 q^{-4}+1+2 q^{-1} \lambda\right) \varepsilon_{\alpha \beta} \varepsilon_{\alpha^{\prime} \beta^{\prime}} M^{\alpha \alpha^{\prime}} M^{\beta \beta^{\prime}} \\
& +k_{2}^{\prime 2}\left(3 q^{-4}+1+2 q^{-1} \lambda\right) \varepsilon_{\dot{\alpha} \dot{\beta}} \varepsilon_{\dot{\alpha}^{\prime} \dot{\beta}^{\prime}} \bar{M}^{\dot{\alpha} \dot{\alpha}^{\prime}} \bar{M}^{\dot{\beta} \dot{\beta}^{\prime}} .
\end{aligned}
$$

From these formulae we can read off the Casimirs of the two $U_{q}(s u(2))$ subalgebras:

$$
\begin{aligned}
& C_{1}+C_{2}=-k_{1}^{\prime 2}\left(3 q^{-4}+1+\lambda_{+}+2 q^{-1} \lambda\right) \varepsilon_{\alpha \beta} \varepsilon_{\alpha^{\prime} \beta^{\prime}} M^{\alpha \alpha^{\prime}} M^{\beta \beta^{\prime}}, \\
& C_{1}-C_{2}=-k_{2}^{\prime 2}\left(3 q^{-4}+1+\lambda_{+}+2 q^{-1} \lambda\right) \varepsilon_{\dot{\alpha} \dot{\beta}} \varepsilon_{\dot{\alpha}^{\prime} \dot{\beta}^{\prime}} \bar{M}^{\dot{\alpha} \dot{\alpha}^{\prime}} \bar{M}^{\dot{\beta} \dot{\beta}^{\prime}} .
\end{aligned}
$$

\subsection{The $q$-deformed Poincaré superalgebra}

Now, we extend the $q$-deformed Poincaré algebra to the $q$-deformed Poincaré superalgebra. To this end, we introduce the supersymmetry generators $Q^{\alpha}$ 
and $\bar{Q}^{\dot{\alpha}}$. These generators carry spinor indices, so they fulfill the well-known quantum plane relations:

$$
\begin{gathered}
Q^{\alpha} Q^{\alpha}=\bar{Q}^{\dot{\alpha}} \bar{Q}^{\dot{\alpha}}=0, \quad \alpha, \dot{\alpha}=1,2, \\
Q^{1} Q^{2}=-q^{-1} Q^{2} Q^{1}, \quad \bar{Q}^{1} \bar{Q}^{2}=-q^{-1} \bar{Q}^{2} \bar{Q}^{1} .
\end{gathered}
$$

Furthermore, they transform as spinor operators under $q$-deformed Lorentz transformations. This observation implies the $q$-commutators

$$
\begin{aligned}
& {\left[V^{\mu \nu}, Q^{\alpha}\right]_{q}=q^{-1} \lambda_{+}^{-1}\left(\sigma^{\mu \nu}\right)_{\beta}^{\alpha} Q^{\beta},} \\
& {\left[V^{\mu \nu}, \bar{Q}^{\dot{\alpha}}\right]_{q}=q^{-1} \lambda_{+}^{-1}\left(\bar{\sigma}^{\mu \nu}\right)_{\dot{\beta}}{ }^{\dot{\alpha}} \bar{Q}^{\dot{\beta}} .}
\end{aligned}
$$

Using Lorentz generators with spinor indices we alternatively have

$$
\begin{gathered}
{\left[M^{\alpha \beta}, Q^{\gamma}\right]_{q}=k_{1}^{-1} q^{-1} \lambda_{+}^{-1} S_{\alpha^{\prime} \beta^{\prime}}^{\alpha \beta} \varepsilon^{\beta^{\prime} \gamma} Q^{\alpha^{\prime}},} \\
{\left[\bar{M}^{\dot{\alpha} \dot{\beta}}, \bar{Q}^{\dot{\gamma}}\right]_{q}=-k_{2}^{-1} q^{-1} \lambda_{+}^{-1} S_{\dot{\alpha}^{\prime} \dot{\beta}^{\prime}}^{\dot{\alpha} \dot{\beta}} \varepsilon^{\dot{\beta}^{\prime} \dot{\gamma}} \bar{Q}^{\dot{\alpha}^{\prime}},} \\
{\left[M^{\alpha \beta}, \bar{Q}^{\dot{\gamma}}\right]_{q}=\left[\bar{M}^{\dot{\alpha} \dot{\beta}}, Q^{\gamma}\right]_{q}=0 .}
\end{gathered}
$$

Next, we turn to the relations between the two supergenerators $Q^{\alpha}$ and $\bar{Q}^{\dot{\alpha}}$. We found

$$
\begin{aligned}
\bar{Q}^{1} Q^{1}+Q^{1} \bar{Q}^{1} & =c q^{-1} P^{-} \\
\bar{Q}^{1} Q^{2}+q^{-1} Q^{2} \bar{Q}^{1} & =-q^{-1} \lambda Q^{1} \bar{Q}^{2}+c q^{-1 / 2} \lambda_{+}^{-1 / 2}\left(P^{3}+q^{-2} P^{0}\right), \\
\bar{Q}^{2} Q^{1}+q^{-1} Q^{1} \bar{Q}^{2} & =-c q^{-3 / 2} \lambda_{+}^{-1 / 2}\left(P^{0}-P^{3}\right), \\
\bar{Q}^{2} Q^{2}+Q^{2} \bar{Q}^{2} & =c q^{-1} P^{+}
\end{aligned}
$$

or, for short,

$$
\left\{\bar{Q}^{\dot{\alpha}}, Q^{\beta}\right\}_{q^{-1}}=c\left(\bar{\sigma}_{\mu}^{-1}\right)^{\dot{\alpha} \beta} P^{\mu},
$$

where the $\sigma_{\mu}^{-1}$ denote Pauli matrices for $q$-deformed Minkowski space (see Ref. [59]). The constant $c$ can be set equal to 1 .

The commutation relations between momentum generators and supergenerators read

$$
\begin{aligned}
P^{+} Q^{1} & =q^{-2} Q^{1} P^{+}, \quad P^{+} Q^{2}=Q^{2} P^{+} \\
P^{0} Q^{1} & =q^{-1} Q^{1} P^{0}, \\
P^{3} Q^{1} & =q^{-1} Q^{1} P^{3}
\end{aligned}
$$




$$
\begin{aligned}
P^{3} Q^{2} & =q^{-1} Q^{2} P^{3}+q^{-3 / 2} \lambda \lambda_{+}^{1 / 2} Q^{1} P^{+}, \\
P^{-} Q^{1} & =Q^{1} P^{-} \\
P^{-} Q^{2} & =q^{-2} Q^{2} P^{-}+q^{-3 / 2} \lambda \lambda_{+}^{1 / 2} Q^{1} P^{3},
\end{aligned}
$$

and

$$
\begin{aligned}
P^{0} \bar{Q}^{1} & =q \bar{Q}^{1} P^{0}, \quad P^{0} \bar{Q}^{2}=q \bar{Q}^{2} P^{0}, \\
P^{-} \bar{Q}^{1} & =\bar{Q}^{1} P^{-}, \quad P^{-} \bar{Q}^{2}=q^{2} \bar{Q}^{1} P^{-} \\
P^{3} \bar{Q}^{1} & =q \bar{Q}^{1} P^{3}-q^{3 / 2} \lambda \lambda_{+}^{1 / 2} \bar{Q}^{2} P^{-} \\
P^{3} \bar{Q}^{2} & =q \bar{Q}^{2} P^{3} \\
P^{+} \bar{Q}^{1} & =q^{2} \bar{Q}^{1} P^{+}-q^{3 / 2} \lambda \lambda_{+}^{1 / 2} \bar{Q}^{2} P^{3}, \\
P^{+} \bar{Q}^{2} & =\bar{Q}^{2} P^{+} .
\end{aligned}
$$

Let us note that in very much the same way as was done in Sec.2.4 the above commutation relations between momentum generators and supergenerators can again be written in terms of $q$-commutators. To this end, the scaling operator appearing in the Hopf structure of the momentum generators has to satisfy

$$
\Lambda Q^{\alpha}=q^{-2} Q^{\alpha} \Lambda, \quad \Lambda \bar{Q}^{\alpha}=q^{-2} \bar{Q}^{\alpha} \Lambda .
$$

Now, we have everything together to write down the $q$-deformed Poincaré superalgebra:

$$
\begin{gathered}
{\left[V^{\mu \nu}, V^{\rho \sigma}\right]_{q}=-q^{-1} \lambda_{+}\left(P_{A}\right)_{\nu^{\prime} \rho^{\prime \prime}}\left(P_{A}\right)^{\rho \sigma}{ }_{\rho^{\prime} \sigma^{\prime}} \eta^{\rho^{\prime \prime} \rho^{\prime}} V^{\nu^{\prime} \sigma^{\prime}},} \\
{\left[V^{\mu \nu}, P^{\rho}\right]_{q}=-q^{-1}\left(P_{A}\right)^{\mu \nu}{ }^{\prime}{ }^{\prime}{ }^{\rho^{\prime} \rho} P^{\nu^{\prime}}} \\
{\left[V^{\mu \nu}, Q^{\alpha}\right]_{q}=q^{-1} \lambda_{+}^{-1}\left(\sigma^{\mu \nu}\right)_{\beta}{ }^{\alpha} Q^{\beta},} \\
{\left[V^{\mu \nu}, \bar{Q}^{\dot{\alpha}}\right]_{q}=q^{-1} \lambda_{+}^{-1}\left(\bar{\sigma}^{\mu \nu}\right)_{\dot{\beta}}^{\dot{\alpha}} \bar{Q}^{\dot{\beta}},} \\
\left(P_{A}\right)^{\mu \nu}{ }_{\mu^{\prime} \nu^{\prime}} P^{\mu^{\prime}} P^{\nu^{\prime}}=0, \\
{\left[P^{\mu}, Q^{\alpha}\right]_{q}=0, \quad\left[P^{\mu}, \bar{Q}^{\dot{\alpha}}\right]_{\bar{q}}=0,} \\
\left\{Q^{\alpha}, Q^{\beta}\right\}_{q}=0, \quad\left\{\bar{Q}^{\dot{\alpha}}, \bar{Q}^{\dot{\beta}}\right\}_{q}=0, \\
\left\{\bar{Q}^{\dot{\alpha}}, Q^{\beta}\right\}_{q^{-1}}=\left(\bar{\sigma}_{\mu}^{-1}\right)^{\dot{\alpha} \beta} P^{\mu} .
\end{gathered}
$$

This algebra is invariant under the conjugation

$$
\begin{gathered}
\overline{P^{\mu}}=\eta_{\mu \nu} P^{\nu}, \quad \overline{Q^{\alpha}}=\varepsilon_{\dot{\alpha} \dot{\beta}} \bar{Q}^{\dot{\beta}}, \quad \overline{Q^{\dot{\alpha}}}=-\varepsilon_{\alpha \beta} Q^{\beta}, \\
\overline{V^{\mu \nu}}=(-1)^{\delta_{\mu 0}+\delta_{\nu 0}} \eta_{\mu \mu^{\prime}} \eta_{\nu \nu^{\prime}} V^{\nu^{\prime} \mu^{\prime}} .
\end{gathered}
$$




\section{Conclusion}

Let us end with some comments on what we have done so far. We considered the $q$-deformed Poincaré algebra and the $q$-deformed Euclidean algebra in three and four dimensions. These algebras describe the symmetry of $q$-deformed Minkowski space and the $q$-deformed Euclidean spaces in three and four dimensions. We extend these algebras to superalgebras by adding two supersymmetry generators with spinor indices. Exploiting consistency arguments we could determine all commutation relations concerning the supersymmetry generators. Furthermore, we were able to write down our $q$-deformed superalgebras in a way that reveals striking similarities to their undeformed counterparts. To achieve this we introduced generators with definite transformation properties and defined their adjoint actions as $q$-commutators.

Lastly, we would like to point out that the $q$-deformed Poincaré superalgebra should be useful in $q$-deforming supersymmetric models. To this end, we reconsider Eq. (131) and contract it with the Pauli matrix $\left(\bar{\sigma}^{\mu}\right)_{\dot{\alpha} \beta}$. In doing so we obtain

$$
\begin{aligned}
P^{\mu} & =\left(\bar{\sigma}^{\mu}\right)_{\dot{\alpha} \beta}\left[\bar{Q}^{\dot{\alpha}} Q^{\beta}+q^{-1} \hat{R}_{\beta^{\prime} \dot{\alpha}^{\prime}} Q^{\beta^{\prime}} \bar{Q}^{\dot{\alpha}^{\prime}}\right] \\
& =\left[\left(\bar{\sigma}^{\mu}\right)_{\dot{\alpha} \beta} \bar{Q}^{\dot{\alpha}} Q^{\beta}+q^{-2}\left(\sigma^{\mu}\right)_{\beta \dot{\alpha}} Q^{\beta} \bar{Q}^{\dot{\alpha}}\right] .
\end{aligned}
$$

Notice that the second equality in (137) holds due to [59]

$$
\left(\sigma^{\mu}\right)_{\beta^{\prime} \dot{\alpha}^{\prime}}=q\left(\bar{\sigma}^{\mu}\right)_{\dot{\alpha} \beta} \hat{R}_{\beta^{\prime} \dot{\alpha}^{\prime}} .
$$

From (137) we get the supersymmetric Hamiltonian

$$
\begin{aligned}
H \equiv P^{0}= & {\left[\left(\bar{\sigma}^{0}\right)_{\dot{\alpha} \beta} \bar{Q}^{\dot{\alpha}} Q^{\beta}+q^{-2}\left(\sigma^{0}\right)_{\beta \dot{\alpha}} Q^{\beta} \bar{Q}^{\dot{\alpha}}\right] } \\
= & {\left[-q^{-1 / 2} \bar{Q}^{1} Q^{2}+q^{1 / 2} \bar{Q}^{2} Q^{1}\right.} \\
& \left.+q^{-5 / 2} Q^{1} \bar{Q}^{2}-q^{-3 / 2} Q^{2} \bar{Q}^{1}\right] .
\end{aligned}
$$

The conjugation properties of $Q^{\alpha}$ and $\bar{Q}^{\dot{\alpha}}$ imply

$$
\bar{H}=\overline{P^{0}}=P^{0}=H,
$$

i.e. our $q$-deformed supersymmetric Hamiltonian is a real operator. It should also be mentioned that $H$ does not commute with the supersymmetry generators, since we have

$$
P^{0} Q^{1}=q^{-1} Q^{1} P^{0}, \quad P^{0} Q^{2}=q^{-1} Q^{2} P^{0},
$$




$$
P^{0} \bar{Q}^{1}=q \bar{Q}^{1} P^{0}, \quad P^{0} \bar{Q}^{2}=q \bar{Q}^{2} P^{0} .
$$

\section{Acknowledgements}

First of all we are very grateful to Eberhard Zeidler for his invitation to the MPI Leipzig, his special interest in our work, and his financial support. Furthermore we would like to thank Fabian Bachmaier and Ina Stein for their support. Finally, we thank Dieter Lüst for kind hospitality.

\section{A Invariant tensors of $q$-deformed quantum spaces}

The aim of this appendix is the following. For the quantum spaces under consideration we list the non-vanishing components of the quantum metric and the totally antisymmetric tensor.

The non-vanishing elements of the two-dimensional spinor metric have the values

$$
\varepsilon^{12}=q^{-1 / 2}, \quad \varepsilon^{21}=-q^{1 / 2} .
$$

The spinor metric is antisymmetric in a $q$-deformed sense and its inverse is given by

$$
\left(\varepsilon^{-1}\right)^{i j}=\varepsilon_{i j}=-\varepsilon^{i j} .
$$

The non-vanishing elements of the three-dimensional Euclidean quantum metric are

$$
g^{+-}=-q, \quad g^{33}=1, \quad g^{-+}=-q^{-1} .
$$

For its inverse $g_{A B}$ we have

$$
g_{A B}=g^{A B} .
$$

The non-vanishing components of the three-dimensional $q$-deformed epsilon tensor take the form

$$
\begin{aligned}
\varepsilon^{-3+} & =-q^{-4}, & & \varepsilon^{3-+}=q^{-2}, \\
\varepsilon^{-+3} & =q^{-2}, & & \varepsilon^{+-3}=-q^{-2}, \\
\varepsilon^{3+-} & =-q^{-2}, & & \varepsilon^{+3-}=1 . \\
\varepsilon^{333} & =-q^{-2} \lambda . & &
\end{aligned}
$$

Next we come to four-dimensional $q$-deformed Euclidean space. Its metric has the non-vanishing components

$$
g^{14}=q^{-1}, \quad g^{23}=g^{32}=1, \quad g^{41}=q .
$$


Its inverse is denoted by $g_{\mu \nu}$ and fulfills

$$
g_{\mu \nu}=g^{\mu \nu}
$$

The non-vanishing components of the epsilon tensor of four-dimensional $q$ deformed Euclidean space are

$$
\begin{array}{lll}
\varepsilon^{1234}=1, & \varepsilon^{1432}=-q^{2}, & \varepsilon^{2413}=-q^{2}, \\
\varepsilon^{2134}=-q, & \varepsilon^{4132}=q^{2}, & \varepsilon^{4213}=q^{3}, \\
\varepsilon^{1324}=-1, & \varepsilon^{3412}=q^{2}, & \varepsilon^{2341}=-q^{2}, \\
\varepsilon^{3124}=q, & \varepsilon^{4312}=-q^{3}, & \varepsilon_{3241}=q^{2}, \\
\varepsilon^{2314}=q^{2}, & \varepsilon^{1243}=-q, & \varepsilon^{2431}=q^{3}, \\
\varepsilon^{3214}=-q^{2}, & \varepsilon^{2143}=q^{2}, & \varepsilon^{4231}=-q^{4}, \\
\varepsilon^{1342}=q, & \varepsilon^{1423}=q^{2}, & \varepsilon^{3421}=-q^{3}, \\
\varepsilon^{3142}=-q^{2}, & \varepsilon^{4123}=-q^{2}, & \varepsilon^{4321}=q^{4},
\end{array}
$$

together with the non-classical components

$$
\varepsilon^{3232}=-\varepsilon^{2323}=-q^{2} \lambda .
$$

The quantum metric of $q$-deformed Minkowski space is given by

$$
\eta^{00}=-1, \quad \eta^{33}=1, \quad \eta^{+-}=-q, \quad \eta^{-+}=-q^{-1},
$$

with inverse

$$
\eta_{\mu \nu}=\eta^{\mu \nu}
$$

As non-vanishing components of the corresponding epsilon tensor we have

$$
\begin{array}{lll}
\varepsilon^{+30-}=1, & \varepsilon^{+-03}=-q^{-2}, & \varepsilon^{3-+0}=q^{-2}, \\
\varepsilon^{3+0-}=-q^{-2}, & \varepsilon^{-+03}=q^{-2}, & \varepsilon^{-3+0}=q^{-4}, \\
\varepsilon^{+03-}=-1, & \varepsilon^{0-+3}=q^{-2}, & \varepsilon^{30-+}=-q^{-2}, \\
\varepsilon^{0+3-}=1, & \varepsilon^{-0+3}=-q^{-2}, & \varepsilon^{03-+}=q^{-2}, \\
\varepsilon^{30+-}=q^{-2}, & \varepsilon^{+3-0}=-1, & \varepsilon^{3-0+}=q^{-2}, \\
\varepsilon^{03+-}=-q^{-2}, & \varepsilon^{3+-0}=q^{-2}, & \varepsilon^{-30+}=-q^{-4}, \\
\varepsilon^{+0-3}=q^{-2}, & \varepsilon^{+-30}=q^{-2}, & \varepsilon^{0-3+}=-q^{-4}, \\
\varepsilon^{0+-3}=-q^{-2}, & \varepsilon^{-+30}=-q^{-2}, & \varepsilon^{-03+}=q^{-4},
\end{array}
$$


and

$$
\begin{aligned}
\varepsilon^{0-0+} & =q^{-3} \lambda, & \varepsilon^{-0+0} & =-q^{-3} \lambda, \\
\varepsilon^{0333} & =-q^{-2} \lambda, & \varepsilon^{3330} & =q^{-2} \lambda, \\
\varepsilon^{3033} & =+q^{-2} \lambda, & \varepsilon^{3030} & =-q^{-2} \lambda, \\
\varepsilon^{3303} & =-q^{-2} \lambda, & \varepsilon^{+0-0} & =-q^{-1} \lambda, \\
\varepsilon^{0303} & =q^{-2} \lambda, & \varepsilon^{0+0-} & =q^{-1} \lambda .
\end{aligned}
$$

Lowering the indices of the epsilon tensor is achieved by the quantum metric. In this manner we have, for example,

$$
\varepsilon_{\mu \nu \rho \sigma}=\eta_{\mu \mu^{\prime}} \eta_{\nu \nu^{\prime}} \eta_{\rho \rho^{\prime}} \eta_{\sigma \sigma^{\prime}} \varepsilon^{\mu^{\prime} \nu^{\prime} \rho^{\prime} \sigma^{\prime}}
$$

\section{References}

[1] H. Miyazawa, Baryon number changing currents, Prog. Theor. Phys. 36 (1966) 1266.

[2] A. Neveu and J. H. Schwarz, Factorizable Dual Model Of Pions, Nucl. Phys. B 31 (1971) 86.

[3] J. L. Gervais and B. Sakita, Field Theory Interpretation of Supergauges in Dual Models, Nucl. Phys. B 34 (1971) 632.

[4] Yu. A. Gol'fand and E. P. Likhtman, Extension of the algebra of Poincaré group generators and violation of P-invariance, Sov. Phys. JETP Lett. 13 (1971) 323.

[5] D. V. Volkov and V. P. Akulov, Possible universal neutrino interaction, Sov. Phys. JETP Lett. 16 (1972) 438.

[6] J. Wess and B. Zumino, Supergauge transformations in four dimensions, Nucl. Phys. B 70 (1974) 39.

[7] J. Wess and J. Bagger, Supersymmetry and Supergravity, Second Edition, Princeton University Press, Princeton (1991).

[8] D. Z. Freedman, P. van Nieuwenhuizen and S. Ferrara, Progress Toward a Theory of Supergravity, Phys. Rev. D 13 (1976) 3214. 
[9] S. Deser and B. Zumino, Consistent Supergravity, Phys. Lett. 62B (1976) 335.

[10] P. P. Kulish and N. Yu. Reshetikin, Quantum linear problem for the Sine-Gordon equation and higher representations, J. Sov. Math. 23 (1983) 2345.

[11] S. L. Woronowicz, Compact matrix pseudogroups, Commun. Math. Phys. 111 (1987) 613.

[12] Yu. I. Manin, Quantum Groups and Non-Commutative Geometry, Centre de Recherche Mathématiques, Montreal (1988).

[13] N. Yu. Reshetikhin, L. A. Takhtadzhyan and L. D. Faddeev, Quantization of Lie Groups and Lie Algebras, Leningrad Math. J. 1 (1990) 193.

[14] M. Takeuchi, Matrix Bialgebras and Quantum Groups, Israel J. Math. 72 (1990) 232.

[15] U. Carow-Watamura, M. Schlieker, M. Scholl and S. Watamura, Tensor Representations of the Quantum Group $S L_{q}(2)$ and Quantum Minkowski Space, Z. Phys. C 48 (1990) 159.

[16] P. Podleś and S. L. Woronowicz, Quantum Deformation of Lorentz Group, Commun. Math. Phys. 130 (1990) 381.

[17] J. Lukierski, A. Nowicki and H. Ruegg, New Quantum Poincaré Algebra and $\kappa$-deformed Field Theory, Phys. Lett. B 293 (1992) 344.

[18] L. Castellani, Differential Calculus on ISO $(N)$, Quantum Poincaré Algebra and q-Gravity, preprint, [hep-th/9312179].

[19] V. K. Dobrev, New q-Minkowski space-time and q-Maxwell equations hierarchy from q-conformal invariance, Phys. Lett. B 341 (1994) 133.

[20] S. Doplicher, K. Fredenhagen and J. E. Roberts, The Quantum Structure of Space-Time at the Planck Scale and Quantum fields, Commun. Math. Phys. 172 (1995) 187.

[21] M. Chaichian and A. P. Demichev, Quantum Poincaré group without dilatation and twisted classical algebra, J. Math. Phys. 36 (1995) 398. 
[22] M. Chaichian, P. P. Kulish, K. Nishijima and A. Tureanu, On a LorentzInvariant Interpretation of Noncommutative Space-Time and its Implications on Noncommutative QFT, Phys. Lett. B 604 (2004) 98, hep-th/0408062.

[23] F. Koch and E. Tsouchnika, Construction of $\theta$-Poincaré Algebras and their invariants on $\mathcal{M}_{\theta}$, Nucl. Phys. B 717 (2005) 387, hep-th/0409012.

[24] M. Fichtenmüller, A. Lorek and J. Wess, q-deformed Phase Space and its Lattice Structure, Z. Phys. C 71 (1996) 533, hep-th/9511106].

[25] B. L. Cerchiai and J. Wess, q-Deformed Minkowski Space based on a q-Lorentz Algebra, Eur. Phys. J. C 5 (1998) 553, math.qa/9801104].

[26] H. Grosse, C. Klimčik and P. Prešnajder, Towards finite quantum field theory in non-commutative geometry, Int. J. Theor. Phys. 35 (1996) 231, hep-th/9505175.

[27] S. Majid, On the q-regularisation, Int. J. Mod. Phys. A 5 (1990) 4689.

[28] R. Oeckl, Braided Quantum Field Theory, Commun. Math. Phys. 217 (2001) 451.

[29] J. Wess, q-deformed Heisenberg Algebras, in H. Gausterer, H. Grosse and L.Pittner, eds., Proceedings of the 38. Internationale Universitätswochen für Kern- und Teilchenphysik, no. 543 in Lect. Notes in Phys., Springer -Verlag, Schladming (2000), math-phy/9910013.

[30] W. Heisenberg, Über die in der Theorie der Elementarteilchen auftretende universelle Länge, Ann. Phys. 32 (1938) 20.

[31] H. Wachter and M. Wohlgenannt, *-Products on quantum spaces, Eur. Phys. J. C 23 (2002) 761, hep-th/0103120.

[32] C. Bauer and H. Wachter, Operator representations on quantum spaces, Eur. Phys. J. C 31 (2003) 261, math-ph/0201023.

[33] H. Wachter, q-Integration on quantum spaces, Eur. Phys. J. C 32 (2004) 281, hep-th/0206083. 
[34] H. Wachter, q-Exponentials on quantum spaces, Eur. Phys. J. C 37 (2004) 379, hep-th/0401113.

[35] D. Mikulovic, A. Schmidt and H. Wachter, Grassmann variables on quantum spaces, Eur. Phys. J. C 45 (2006) 529, [hep-th/0407273].

[36] A. Schmidt and H. Wachter, Superanalysis on quantum spaces, JHEP 0601 (2006) 84, hep-th/0411180].

[37] H. Wachter, Analysis on q-deformed quantum spaces, Int. J. Mod. Phys. A 22 (2007) 95.

[38] J. Wess and B. Zumino, Covariant differential calculus on the quantum hyperplane, Nucl. Phys. B. Suppl. 18 (1991) 302.

[39] S. Majid, Examples of braided groups and braided matrices, J. Math. Phys. 32 (1991) 3246.

[40] U. Carow-Watamura, M. Schlieker and S. Watamura, $S O_{q}(N)$-covariant differential calculus on quantum space and deformation of Schrödinger equation, Z. Phys. C 49 (1991) 439.

[41] A. Lorek, W. Weich and J. Wess, Non-commutative Euclidean and Minkowski Structures, Z. Phys. C 76 (1997) 375, q-alg/9702025.

[42] V. G. Drinfeld, Hopf algebras and the quantum Yang-Baxter equation, Sov. Math. Dokl. 32 (1985) 254.

[43] M. Jimbo, A q-analogue of $U(g)$ and the Yang-Baxter equation, Lett. Math. Phys. 10 (1985) 63.

[44] V. G. Drinfeld, Quantum groups, in A. M. Gleason, ed., Proceedings of the International Congress of Mathematicians, Amer. Math. Soc., 798 (1986).

[45] W. B. Schmidke, J. Wess and B. Zumino, A q-deformed Lorentz Algebra in Minkowski phase space, Z. Phys. C 52 (1991) 471.

[46] O. Ogievetsky, W. B. Schmidke, J. Wess and B. Zumino, q-Deformed Poincaré Algebra, Commun. Math. Phys. 150 (1992) 495. 
[47] S. Majid, Braided momentum in the q-Poincaré group, J. Math. Phys. 34 (1993) 2045.

[48] Yu. I. Manin, Multiparametric Quantum Deformation of General Linear Supergroup, Comm. Math. Phys. 123 (1989) 163.

[49] T. Kobayashi and T. Uematsu, q-Deformed Superconformal Algebra on Quantum Superspace, Phys. Lett. B 306 (1993) 27, hep-th/9302064.

[50] V. Lyubashenko and A. Sudbery, Quantum supergroups of $G L(n \mid m)$ type, Duke Math. J. 90 (1997).

[51] S. Majid and M. J. Rodríguez-Plaza, Non-standard Quantum Groups and Superization, J. Math. Phys. 36 (1995) 7081 , q-alg/9506015.

[52] S. Ferrara, M. Lledo and O. Macia, Supersymmetry in noncommutative superspaces, JHEP 0309 (2003) 68, [hep-th/0307039].

[53] D. Mikulovic, Seiberg-Witten map for superfields on canonically deformed $N=1, d=4$ superspace, JHEP 01 (2004) 63, hep-th/0310065.

[54] M. C. Witt, Deformierter Superraum und Diskussion zu einer deformierten Supersymmetriealgebra, Ph.D. thesis, Ludwig-MaximiliansUniversität München, Fakultät für Physik (1998).

[55] P. Kosiński, J. Lukierski, P. Maślanka and J. Sobczyk, The classical basis for $\kappa$-deformed Poincaré (super)algebra and the second $\kappa$ deformed supersymmetric Casimir, Mod. Phys. Lett. A 10 (1995) 2599, hep-th/9412114.

[56] P. Kosiński, J. Lukierski, P. Maślanka and J. Sobczyk, Quantum Deformation of the Poincaré Supergroup and $\kappa$-deformed Superspace, J. Phys. A 27 (1994) 6827, hep-th/9405076.

[57] P. Kosiński, J. Lukierski, P. Maślanka and J. Sobczyk, $\kappa$-Deformation of Poincaré Superalgebra with Classical Lorentz Subalgebra and its Graded Bicrossproduct Structure, J. Phys. A 28 (1995) 2255, [hep-th/9411115].

[58] S. Wolfram, The Mathematica Book, fourth ed., University Press, Cambridge (1999). 
[59] A. Schmidt and H. Wachter, Spinor calculus for q-deformed quantum spaces I, preprint, [hep-th/0705.1640].

[60] A. Schmidt and H. Wachter', q-Deformed quantum Lie algebras, J. Geom. Phys. 56 (2006), mat-ph/0500932.

[61] S. Majid, Foundations of Quantum Group Theory, University Press, Cambridge (1995).

[62] M. Chaichian and A. P. Demichev, Introduction to Quantum Groups, World Scientific, Singapore (1996).

[63] A. Klimyk and K. Schmüdgen, Quantum Groups and their Representations, Springer Verlag, Berlin (1997).

[64] P. Schupp, P. Watts and B. Zumino, Bicovariant Quantum Algebras and Quantum Lie algebras, Commun. Math. Phys. 157 (1993) 305.

[65] A. Sudbery, Quantum differential calculus and lie algebras, Int. J. Mod. Phys. A (1993) 228.

[66] L. C. Biedenharn and M. Tartini, On q-Tensor Operators for Quantum Groups, Lett. Math. Phys. 20 (1990) 271.

[67] S. Majid, Quantum and Braided Lie algebras, J. Geom. Phys. 13 (1994) 307.

[68] H. Ocampo, $S O_{q}(4)$ quantum mechanics, Z. Phys. C 70 (1996) 525.

[69] C. Blohmann, Spin Representations of the q-Poincaré Algebra, Ph.D. thesis, Ludwig-Maximilians-Universität München, Fakultät für Physik (2001).

[70] F. Bachmaier, The free particle on q-Minkowski space, Doktorarbeit, Ph.D. thesis, Ludwig-Maximilians-Universität München, Fakultät für Physik (2003). 